\title{
Effectiveness of Supporting Informal Caregivers of People with Dementia: A Systematic Review of Randomized and Non-Randomized Controlled Trials
}

\author{
Sophie Vandepitte ${ }^{\mathrm{a}, *}$, Nele Van Den Noortgate ${ }^{\mathrm{b}}$, Koen Putman $^{\mathrm{c}}$, Sofie Verhaeghe ${ }^{\mathrm{a}}$, \\ Kristof Faes ${ }^{\mathrm{a}}$ and Lieven Annemans ${ }^{\mathrm{a}}$ \\ ${ }^{a}$ Faculty of Medicine and Health Sciences, Department of Public Health, Ghent University, Belgium \\ ${ }^{\mathrm{b}}$ Faculty of Medicine and Health Sciences, Department of Internal Medicine, Ghent University, \\ Brussels, Belgium \\ ${ }^{\mathrm{c}}$ Faculty of Medicine and Pharmacy, Department of Medical Sociology, Vrije Universiteit Brussel, \\ Brussels, Belgium
}

Accepted 17 February 2016

\begin{abstract}
.
Background: Dementia is known as a major public health problem affecting both patients and caregivers, and placing a high financial strain upon society. In community-dwelling patients, it is important to support informal caregivers in order to help them sustain their demanding role. Previous reviews about effectiveness of such supporting strategies often included a small number of studies, focused only on particular supportive types, particular outcomes, or solely on caregivers.

Objective: A general systematic review was conducted investigating effectiveness of different supportive strategies on at least the well-being of the caregiver or the care-recipient.

Methods: A systematic literature search was conducted in Web of Science and PubMed. An adapted version of the Downs and Black (1998) checklist was used to assess methodological quality. A new classification was developed to group different types of caregiver support.

Results: Fifty-three papers met the inclusion criteria. Although $87 \%$ of the interventions were to some extent effective, methods and findings were rather inconsistent. Psychoeducational interventions generally lead to positive outcomes for caregivers, and delay permanent institutionalization of care-recipients. Cognitive behavioral therapy decreases dysfunctional thoughts among caregivers. Occupational therapy decreases behavioral problems among patients and improves self-efficacy of caregivers. In general, those interventions tailored on individual level generate better outcomes. Comparative research on respite care was very rare.

Conclusions: Despite methodological inconsistency, supporting caregivers appears to be an effective strategy often improving well-being of caregiver or care-recipient and resulting in additional benefits for society. However, there is a need for more research on the (cost)-effectiveness of respite care.
\end{abstract}

Keywords: Alzheimer's disease, caregiver, dementia, effectiveness, support

\footnotetext{
${ }^{*}$ Correspondence to: Sophie Vandepitte, MA, Ghent University, De Pintelaan 185, Ghent 9000, Belgium. Tel.: +32 0 93328360; Fax: +32 0 93324994; E-mail: Sophie.Vandepitte@ UGent.be.
} 


\section{INTRODUCTION}

Dementia is known as a major public health problem with serious physical and emotional consequences for patients and their caregivers and a high financial strain upon society. Most people with dementia live at home and are cared for by informal caregivers, mostly spouses and children, who play a crucial role in the supervision and therapy because of the progressive mental decline of the disease. Knowing that on the one hand there is a high cost of the disease for the society due to frequent hospitalization and permanent institutionalization in nursing homes and, on the other hand, people with dementia prefer to remain in their trusted environment as long as possible, it can be stated that informal caregivers are probably cost saving for society, but also essential in the care process [1-9]. Especially given the emerging focus on person-centered care, caregivers are indispensable to maximize well-being of people with dementia since they are best placed to identify their unique needs and desires [10].

Although caregiving for a loved one can be very satisfying, it also demands a lot from informal caregivers over long periods [5, 11]. Most research in the past decade has shown that informal caregivers have more health problems, visit healthcare professionals more frequently, suffer from isolation, and have an increased risk of depression, distress, and other illness [1, 2, 6, 11-15]. Because caregiver burden can reduce quality of caregiving and increases the likelihood of care-recipient placement into institutional care, there is also a substantial risk for the care-recipient $[6,16,17]$.

To prevent caregivers from getting overburdened different supportive interventions, such as psychoeducation, respite care, cognitive behavioral interventions, and occupational therapy, have been developed to improve their well-being. Although well-being remains a non-uniform concept with various definitions, in this study we defined wellbeing as an evaluation of a person's quality of life, life satisfaction, or positive affect. It refers to a balance between the social, physical, and psychological resources caregivers need in order to meet their social, psychological, and physical challenges [18-21]. Although many interventions demonstrated positive impacts on different outcomes such as burden [22, 23], quality of life of the caregiver [24], and delay in hospitalization [25]; other similar studies did not find the same benefits [26-28]. Inconsistency of the current findings can be explained by the methodological issues caused by the complex nature of this type of research, e.g., [4, 29-35], such as: different duration of interventions, different intensity and characteristics of interventions, and the variety of caregivers and care-recipients characteristics. Additionally, these interventions often suffer from insufficient statistical power, short follow-up periods, and the use of various instruments to measure outcomes. To give caregivers of people with dementia the care they need, more insight is still needed in this area $[1,11,36]$.

After a review of the existing reviews in this field (Supplementary Table 1), we concluded that there was a need for an updated general systematic review for different reasons. First, most of the existing reviews about supportive interventions targeting informal caregivers have focused either on a particular type of intervention: for example internet based interventions [2] or respite care [37-39]; on a particular outcome: such as anxiety [40], quality of life [41], delay in institutionalization [42], or burden [43]; or only on the impact for the caregiver [2, 44, 45] ignoring other possible effects on the care-recipient or vice versa. Second, many reviews excluded respite care as a type of caregiver support while this should also be recognized as a supportive strategy for caregivers. Third, some reviews only included a small number of studies potentially missing important evidence of non-included but eligible interventions [29]. Finally, many reviews included both controlled and uncontrolled studies making comparison between interventions difficult $[12,45]$.

A general systematic review is thus desirable, only including recent studies with both an intervention arm and a control arm, taking different types of interventions targeting caregivers into account, and measuring outcome effects at least on the caregiver or/and on the care-recipient. The last review which met the abovementioned criteria was published five years ago and only included randomized controlled trials through 2008 [33]. It is to be expected that since then, there is new evidence regarding effectiveness of supportive strategies for informal caregivers.

\section{Aim of this systematic review}

The current review aims to address the following question: Are initiatives to support informal caregivers of people with dementia effective for caregivers and/or care-recipients in comparison to standard dementia care? More specifically: what impact does supporting informal caregivers of people 
with dementia have on the well-being of those caregivers and/or on the well-being of people with dementia?

\section{METHODS}

\section{Search strategy}

A systematic literature search was conducted in PubMed and Web of Science between 15 February 2015 and 12 March 2015. PubMed was used as a core database first identifying potential 'MeSH Terms' which were then included in the search strings to fully capture all relevant synonyms. Used terms in the search strings were: effectiveness*, impact*, effect*, efficacy*, support*, intervention studies $(\mathrm{MeSH})^{*}$, intervention*, program*, programme*, therapy*, psychoeducation*, psycho-education*, occupational therapy $(\mathrm{MeSH})^{*}$, cognitive behavioral therapy $(\mathrm{MeSH})^{*}$, respite*, respite care $(\mathrm{MeSH})^{*}$, day care $(\mathrm{MeSH})^{*}$, day-care*, partial hospitalization*, intermediate care facilities (MeSH), intermediate care*, temporary admission*, short stay*, residential respite*, night care $(\mathrm{MeSH})^{*}$, night-time care*, in-home respite*, home respite*, host family respite*, caregiver*, carer*, care giver*, spouse caregiver*, family caregiver*, informal care*, informal caregiver*, dementia (MeSH)*, Alzheimer disease $(\mathrm{MeSH})^{*}$, Alzheimer*, Alzheimer's disease*, and frail elderly (MeSH)*.

\section{Study selection}

The inclusion and exclusion criteria used in this systematic review are represented in Fig. 1.

\section{Data extraction}

The initial search in two databases resulted in a total of 1,033 studies. After eliminating duplicates, 881 studies remained. The remaining studies were further analyzed based on reviewing titles and abstracts resulting in 88 studies assessed for eligibility. After reading full texts of those papers, 53 studies were finally included in this systematic review. Figure 2 illustrates the data extraction process.

The first 50 abstracts assessed for eligibility were double-checked by two researchers to avoid bias. When there was disagreement about eligibility, it was discussed between the researchers. Mostly disagreement occurred when it was not clear if inclusion criteria had been met based on reviewing the abstract. In that case, the full text was read and consensus achieved.

\section{Quality appraisal}

Study quality was fully assessed by one reviewer using the 27-items checklist for the assessment of the methodological quality for both randomized and non-randomized studies of health care interventions created by Downs and Black [46]. A detailed list of the scores per item per study can be obtained upon request (Supplementary Table 2). To avoid bias, a second reviewer assessed quality of ten randomly selected papers. When disagreement occurred this was discussed between the two reviewers. If no consensus could be reached, a third reviewer was consulted. Based on the obtained consensus regarding the 10 randomly selected papers the first reviewer followed a consistent approach to assess the rest of the papers.

\begin{tabular}{|c|c|}
\hline Inclusion criteria & Exclusion criteria \\
\hline The study had to report about effectiveness of supportive psychosocial strategies. & Interventions concerning remuneration of informal care were not included in this review. \\
\hline $\begin{array}{l}\text { The intervention had to target informal caregivers. Dyad interventions targeting both caregiver } \\
\text { and care-recipient were also allowed for inclusion. }\end{array}$ & Interventions only focusing on the care-recipients were excluded. \\
\hline $\begin{array}{l}\text { Caregivers had to be informal meaning that they should not be paid caregivers or health } \\
\text { workers in the caregiving role under investigation. }\end{array}$ & $\begin{array}{l}\text { When studies only included caregivers with specific psychological or physical morbidity, such } \\
\text { as anxiety or depression at baseline, they were excluded. }\end{array}$ \\
\hline \multicolumn{2}{|l|}{$\begin{array}{l}\text { The care-recipient had to be diagnosed with dementia based on one of the existing diagnostic } \\
\text { criteria such as the DSM (Diagnostic and Statistical Manual of Mental Disorders). There was } \\
\text { no restriction on different types or stages of dementia. Studies addressing frail elderly were } \\
\text { also included if people with dementia were discussed in the study. }\end{array}$} \\
\hline The care-recipient had to live in the community. & $\begin{array}{l}\text { Studies including care-recipients who were definitively placed in residential care were } \\
\text { excluded. }\end{array}$ \\
\hline $\begin{array}{l}\text { The study design had to include a control group receiving treatment as usual or no treatment at } \\
\text { all. Studies could thus be randomized controlled trials, quasi-experimental studies or pre- } \\
\text { posttest studies with control group. }\end{array}$ & $\begin{array}{l}\text { Qualitative research, studies without control group, case studies, systematic reviews, and meta- } \\
\text { analyses were excluded. }\end{array}$ \\
\hline \multicolumn{2}{|l|}{$\begin{array}{l}\text { At least one of the following outcomes had to be quantitatively measured: impact on the well- } \\
\text { being of the caregiver or impact on the well-being of the care-recipient. }\end{array}$} \\
\hline $\begin{array}{l}\text { The studies had to be conducted in Europe, Nord America or Oceania (Australia or New } \\
\text { Zealand), be written in English, and be published in the year } 2000 \text { or later. }\end{array}$ & \\
\hline
\end{tabular}

Fig. 1. Inclusion and exclusion criteria of this review. 


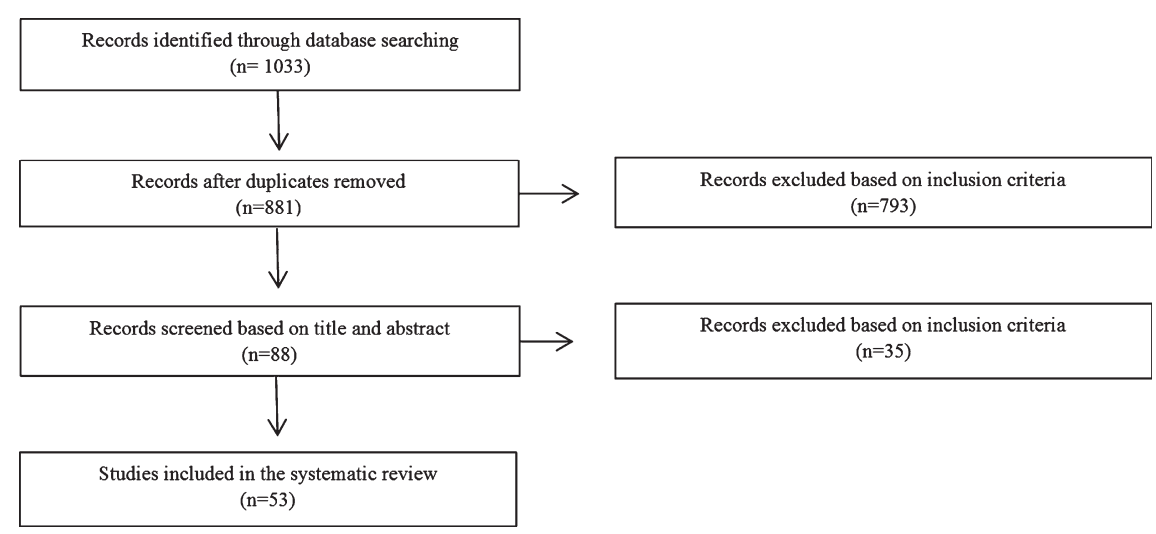

Fig. 2. Flowchart of the data extraction process.

In line with McKechnie et al., some items from the original checklist (related to adverse events; representativeness of staff, places, and facilities; blinding study subjects; and compliance) were not applied because of not relevant to the investigated papers [44]. Finally, the item that deals with statistical power was simplified to a choice of scoring either zero or one point, in which one point means that the study had sufficient power to detect a clinical effect [47]. Given these adjustments each study could now reach a maximum score of 23. Scores from 0-7 indicate very low methodological quality, 8-13 low methodological quality, 14-18 medium methodological quality, and 19-23 high methodological quality.

\section{Study classification}

Different types of interventions can be defined but the overlap of common components in many interventions makes it difficult to create an distinct classification [15]. As a result, various classifications are used in the existing literature. In this systematic review, five main types of support for caregivers were distinguished: psychoeducational interventions, respite care, occupational therapy interventions, cognitive behavioral interventions, and miscellaneous interventions. In accordance to Pinquart \& Sorensen, when a certain intervention contained components of different distinguished support types, they were classified in one of the first four categories based on their dominant component [12]. When no dominant strategy could be identified, the intervention was classified under miscellaneous interventions. In the following paragraphs, each main type of support, except miscellaneous interventions (see above) is explained.

Psychoeducational interventions are the most common used type of intervention to support caregivers of people with dementia with a strong emphasis on gaining knowledge, on developing skills to deal with disease-related problems, and on providing social support. Typically these are multicomponent containing a broad range of activities such as education, social support, counseling, problem-solving techniques, coping, and skill building activities [31, $48,49]$. When psychoeducational interventions were solely focusing on one strategy or contained multiple activities, they were respectively further classified as single or multicomponent interventions.

Second, respite care can be defined as services providing a temporary break to caregivers and can be further distinguished into: community-based respite care (including in-home respite $\&$ host family respite) and residential respite care (including day care, temporary residential admission, and night-time care) $[38,50]$.

Occupational therapy interventions are originally developed to maintain and improve function, independence, and participation in activities of daily living in people with illness, injury or disability by performing meaningful activities. When occupational therapy targets caregivers the purpose is to reduce burden of the caregivers by increasing their ability to handle problematic behaviors and their sense of competence [51, 52].

Cognitive-behavioral therapy is a type of specialized mental health counseling including both behavioral and cognitive techniques. The aim of these structured interventions is to help caregivers be aware of automatic, dysfunctional thoughts and to see problematic situations more clearly so they can respond more effectively in the future. Cognitivebehavioral therapy interventions often partly overlap psychoeducational interventions, especially when coping-based strategies and skills training are used 
in psychoeducational interventions, but must nevertheless be classified as a separate type. The latter by its specialized structured character based on cognitive components with a strong emphasis on cognitive processes as a mediator for behavioral changes [53, 54].

Finally, each type of support except respite care is once more divided into individual based, group based or a combination of both depending on whether they are delivered at individual level, group level or a combination of both. Figure 3 illustrates the developed classification for this review.

\section{RESULTS}

In this chapter, the results of all 53 included studies are discussed by intervention type. Possible outcomes investigated in the included interventions for informal caregivers are related to quality of life/well-being, mental health, physical health, competence/selfefficacy, burden, attitudes, and resources.

More detailed information on the included studies is displayed in Table 1. When stated that a particular intervention had an effect, this means that there was a significant positive effect on at least one outcome measure at $p \leq 0.05$ in the intervention group compared to control group. This effect could be found in either the primary outcome(s), the secondary outcome(s), in a subscale of one of the outcome measures, or in a subgroup of participants. The primary outcomes are mentioned in the results and additionally in Table 1 whenever this was clear stipulated in the studies considered.

\section{Psychoeducational interventions}

A total of 37 psychoeducational papers (11 of high, 23 of medium, and three of low methodological quality), of which 32 showed positive effects
(86\%), were identified. In general, psychoeducational interventions generated more often benefits for caregivers ( $83 \%$ of the interventions investigating informal caregiver outcomes) than for care-recipients (39\% of the interventions investigating care-recipient outcomes). The multicomponent psychoeducational interventions seem to be most popular generating better results $(90 \%)$ than the single component psychoeducational interventions $(67 \%)$. In general the most frequently mentioned benefits are found in selfefficacy, depressive symptoms and burden scales of caregivers, and to a less extent in delaying nursing home placement of care-recipients.

To structure the results about psychoeducational interventions and in order to better compare those interventions, we grouped the psychoeducational interventions based on the different supportive strategies they used, such as: support group, education, training, and counseling.

\section{Single component interventions}

Four of the six included single component interventions (67\% were effective) showed some small benefits. Based on the performed quality appraisal, three studies were considered to be of high methodological quality and the three others of medium methodological quality.

\section{Individual based single component interventions}

Two of the three individual delivered single component psychoeducational interventions showed small benefits for caregivers, mostly only for certain caregiver subgroups. One study showed benefits for care-recipients.

One intervention of high methodological quality consisted of a care management program to support

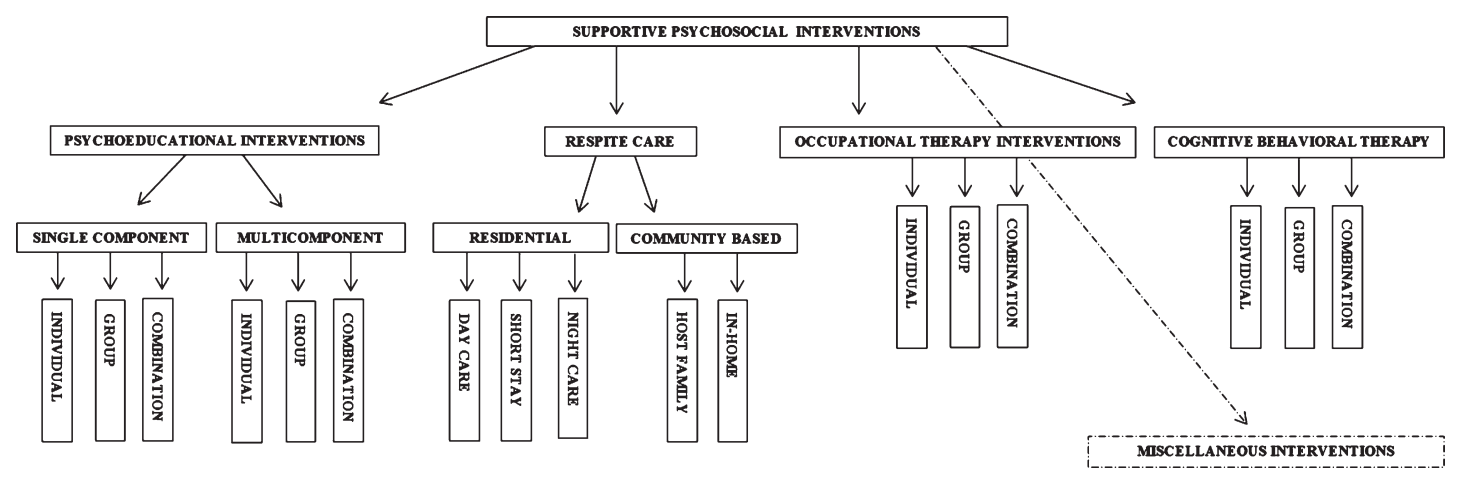

Fig. 3. Classification of supportive interventions for caregivers of people with dementia. 


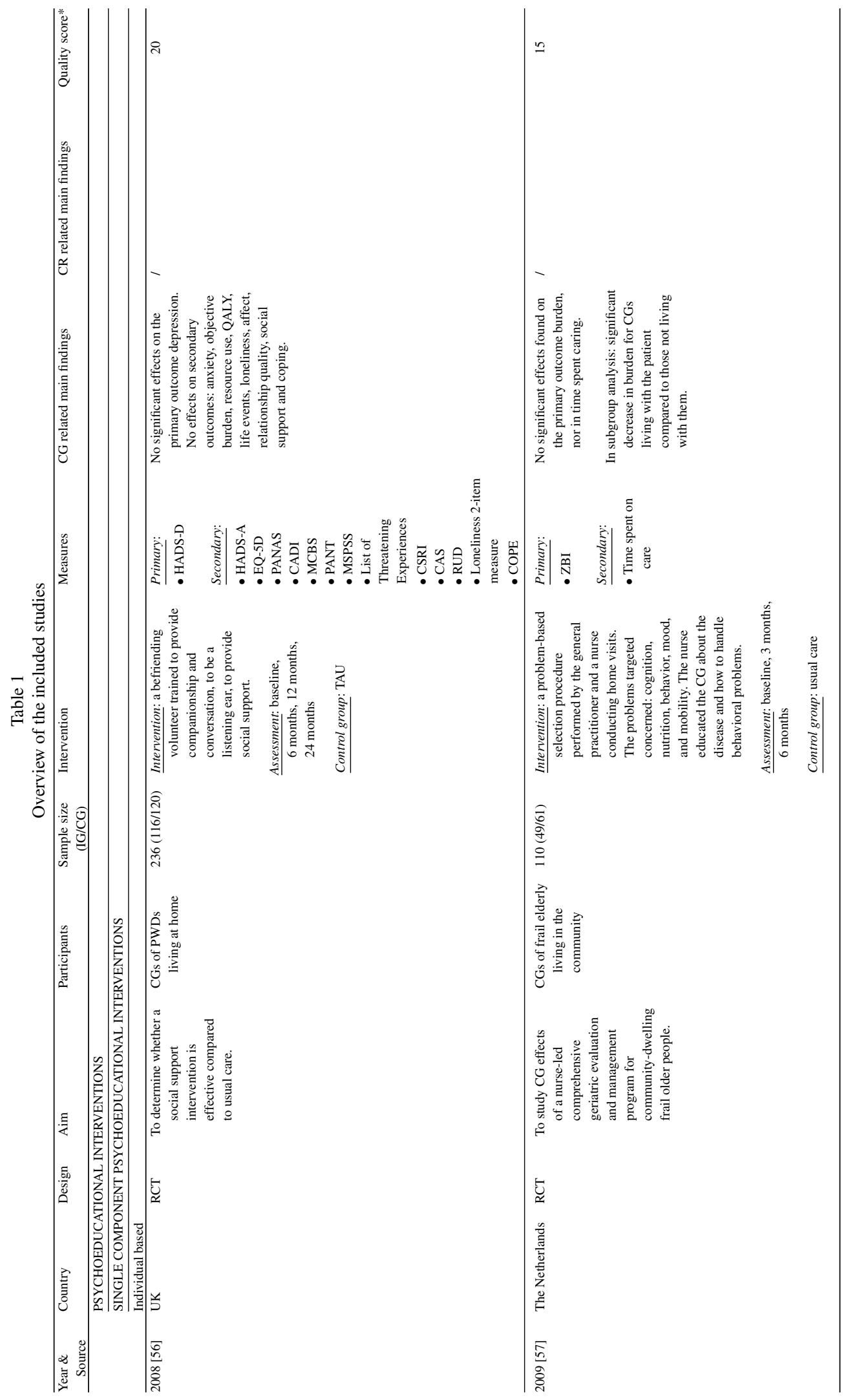




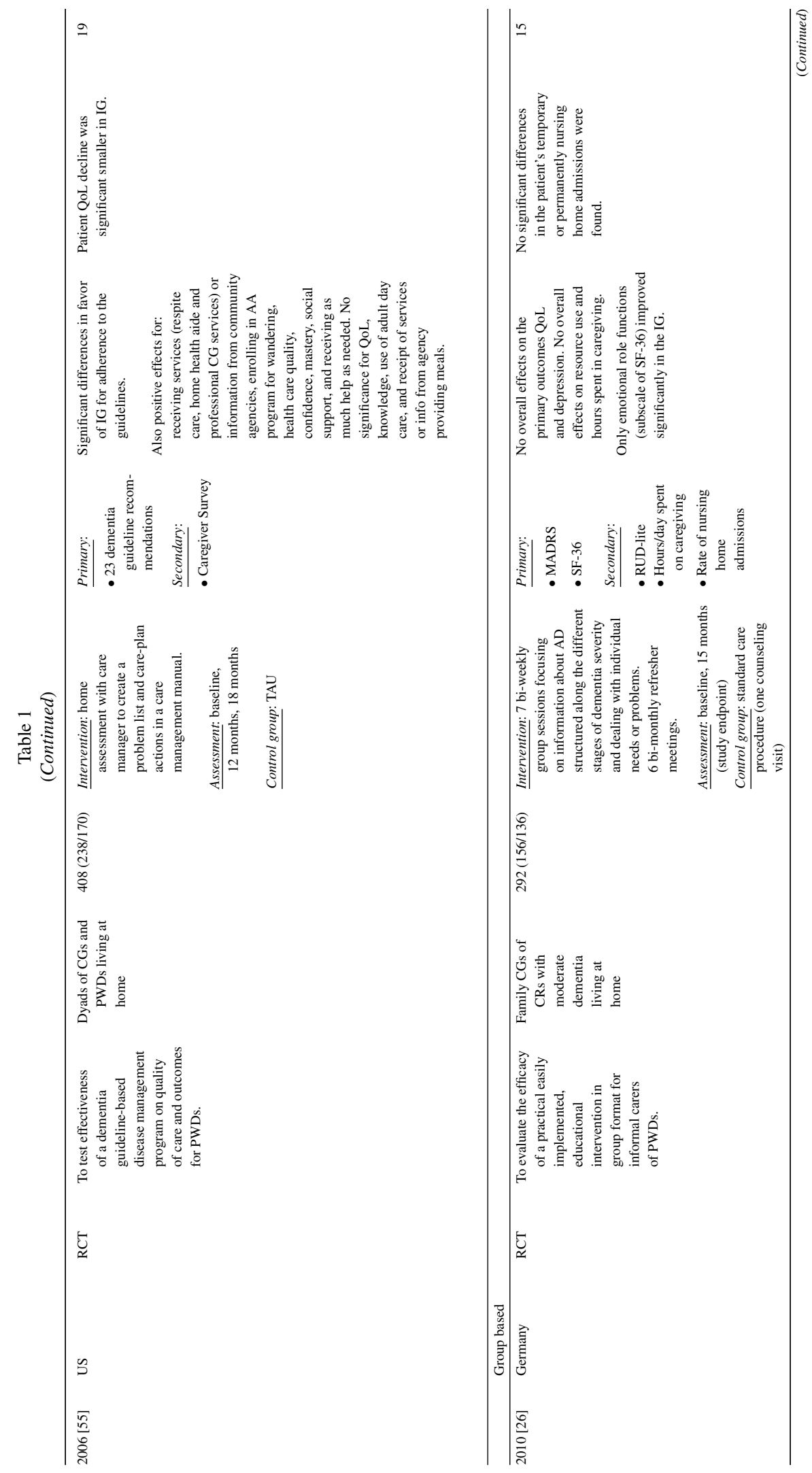



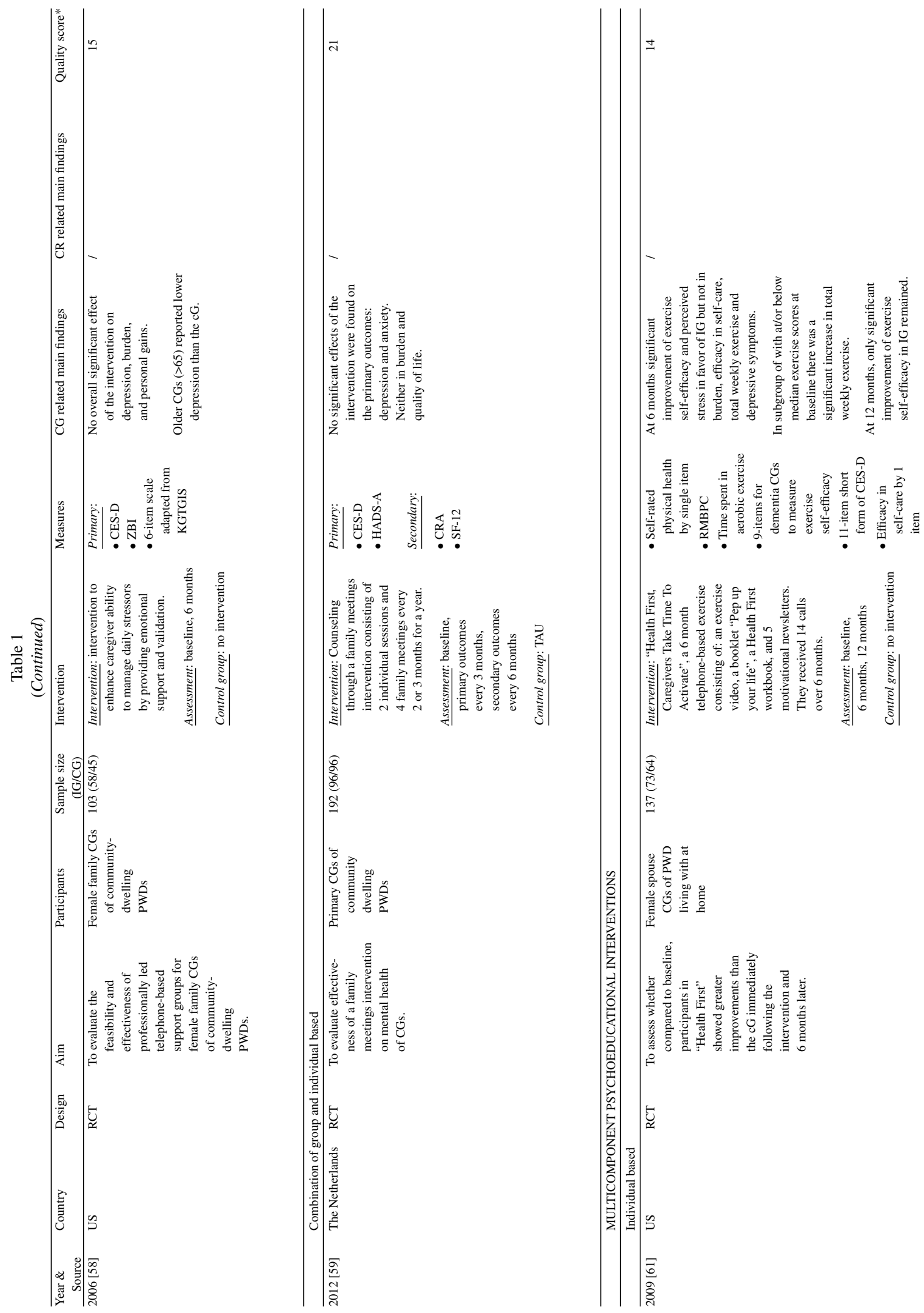


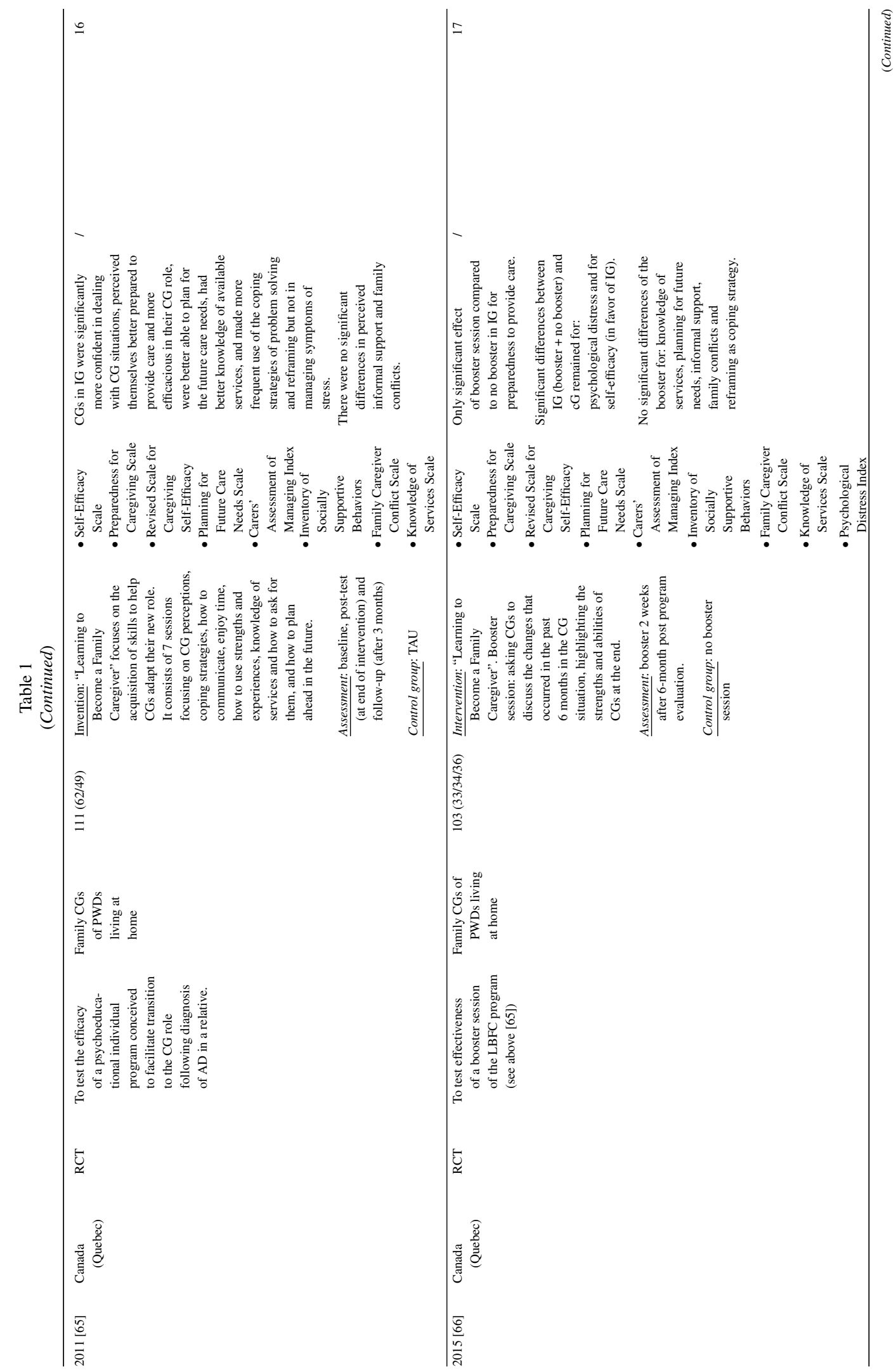




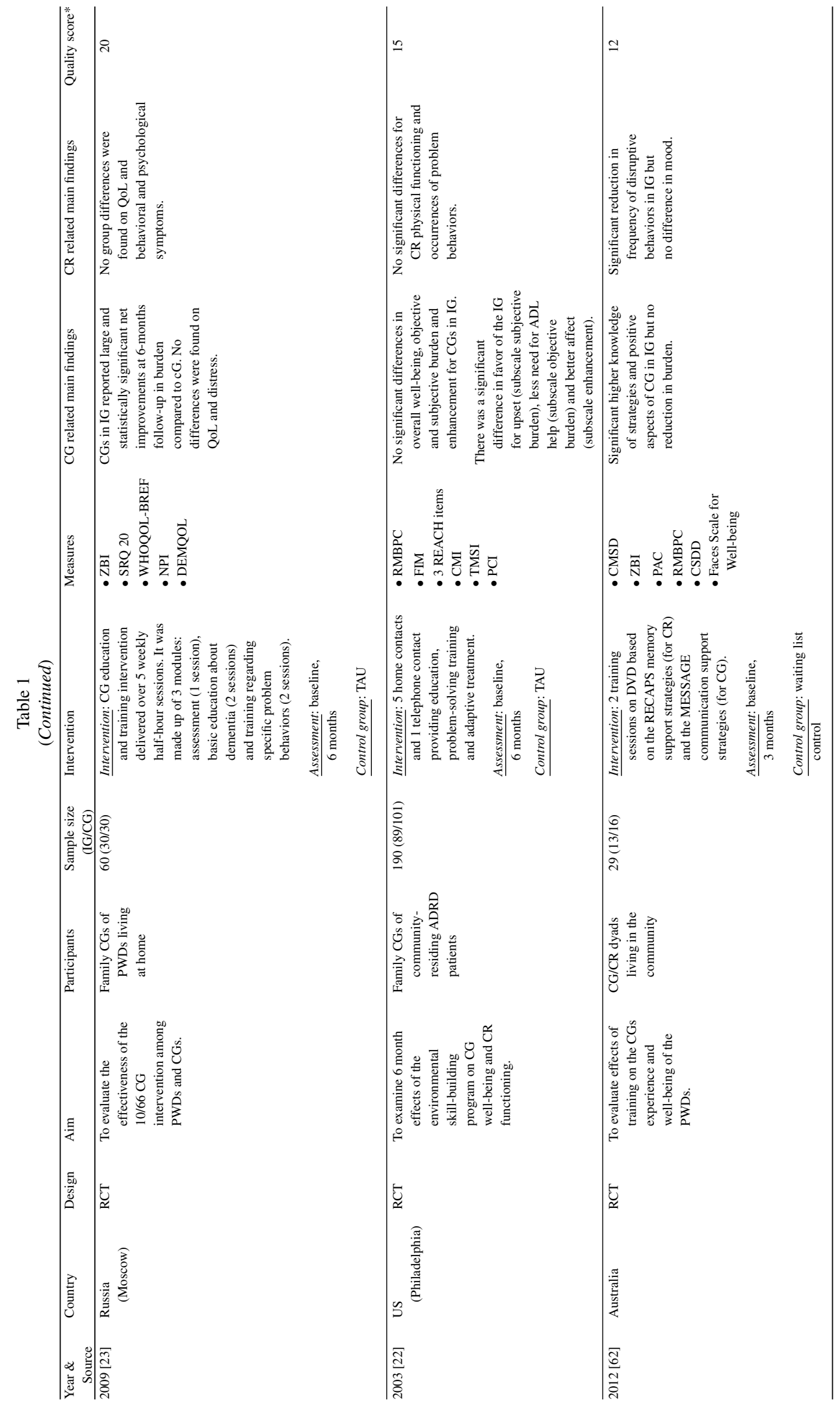




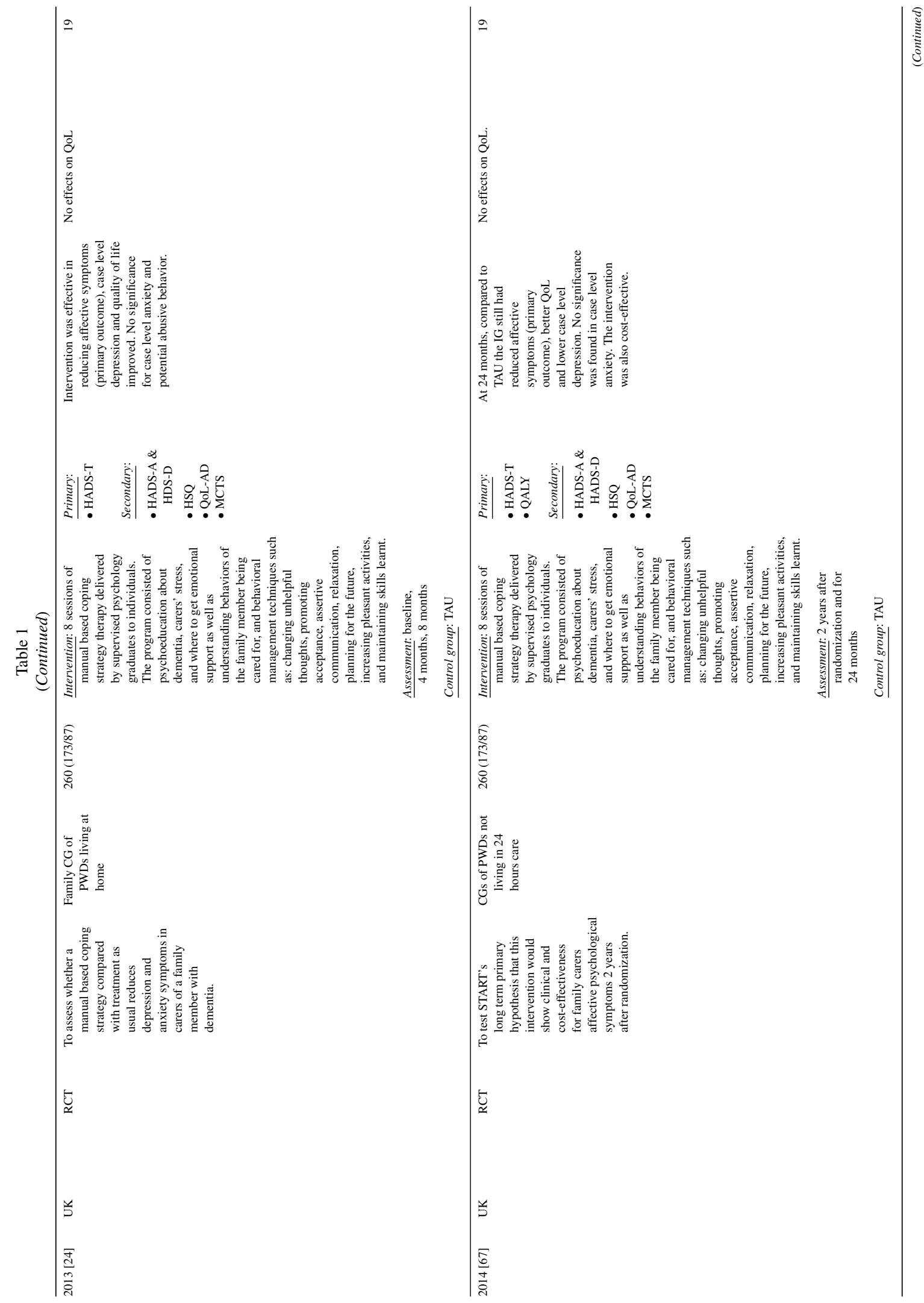




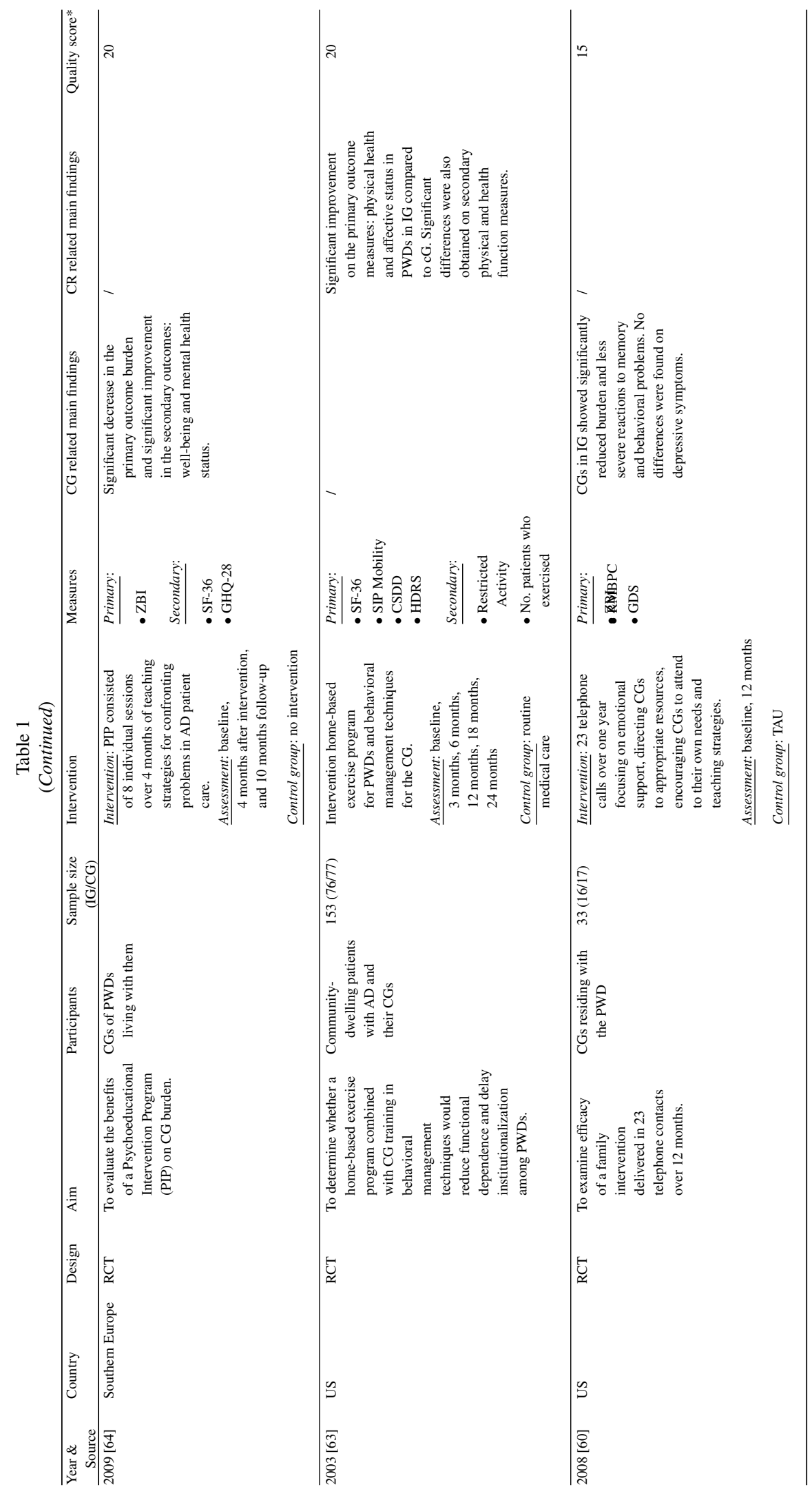




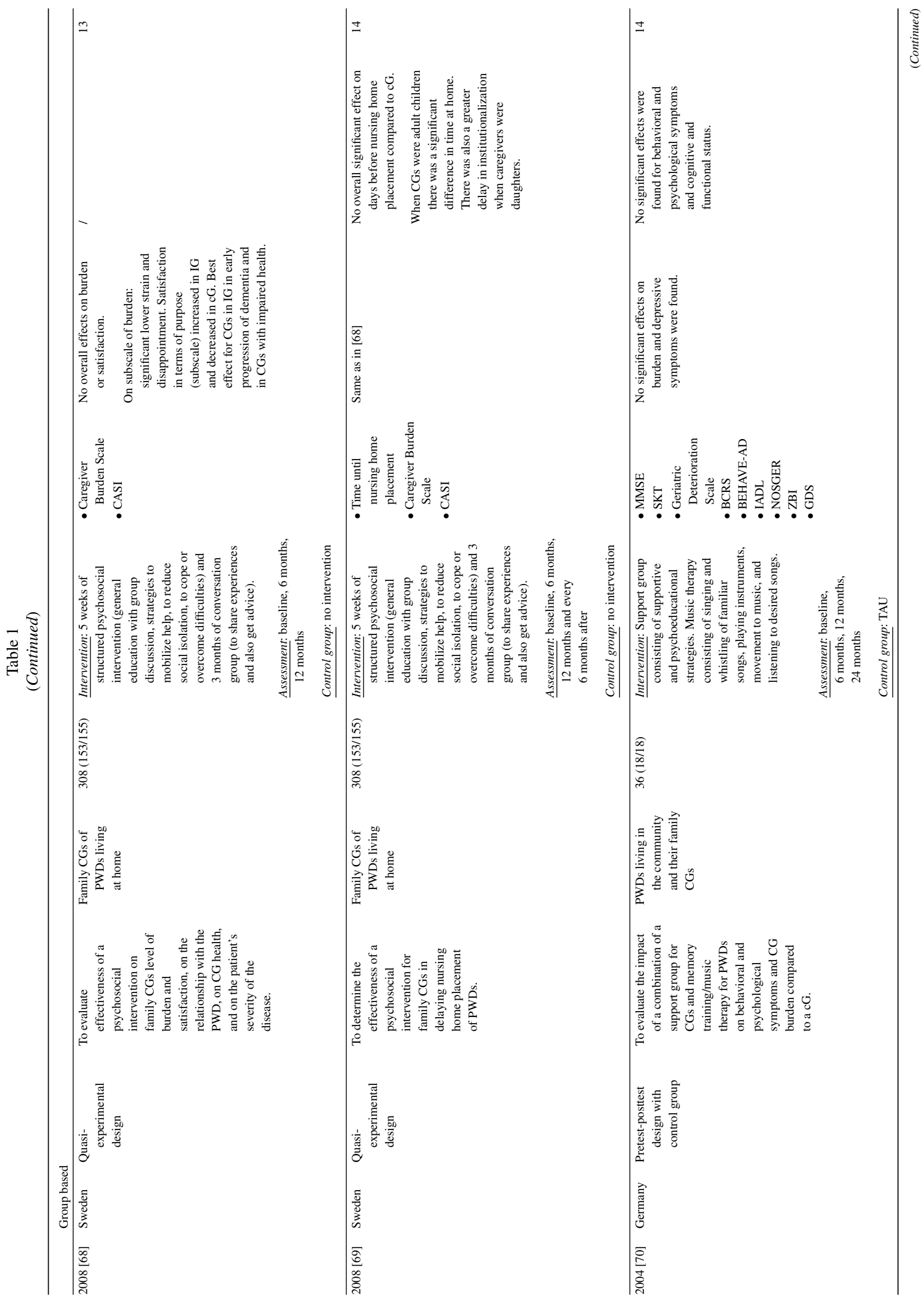




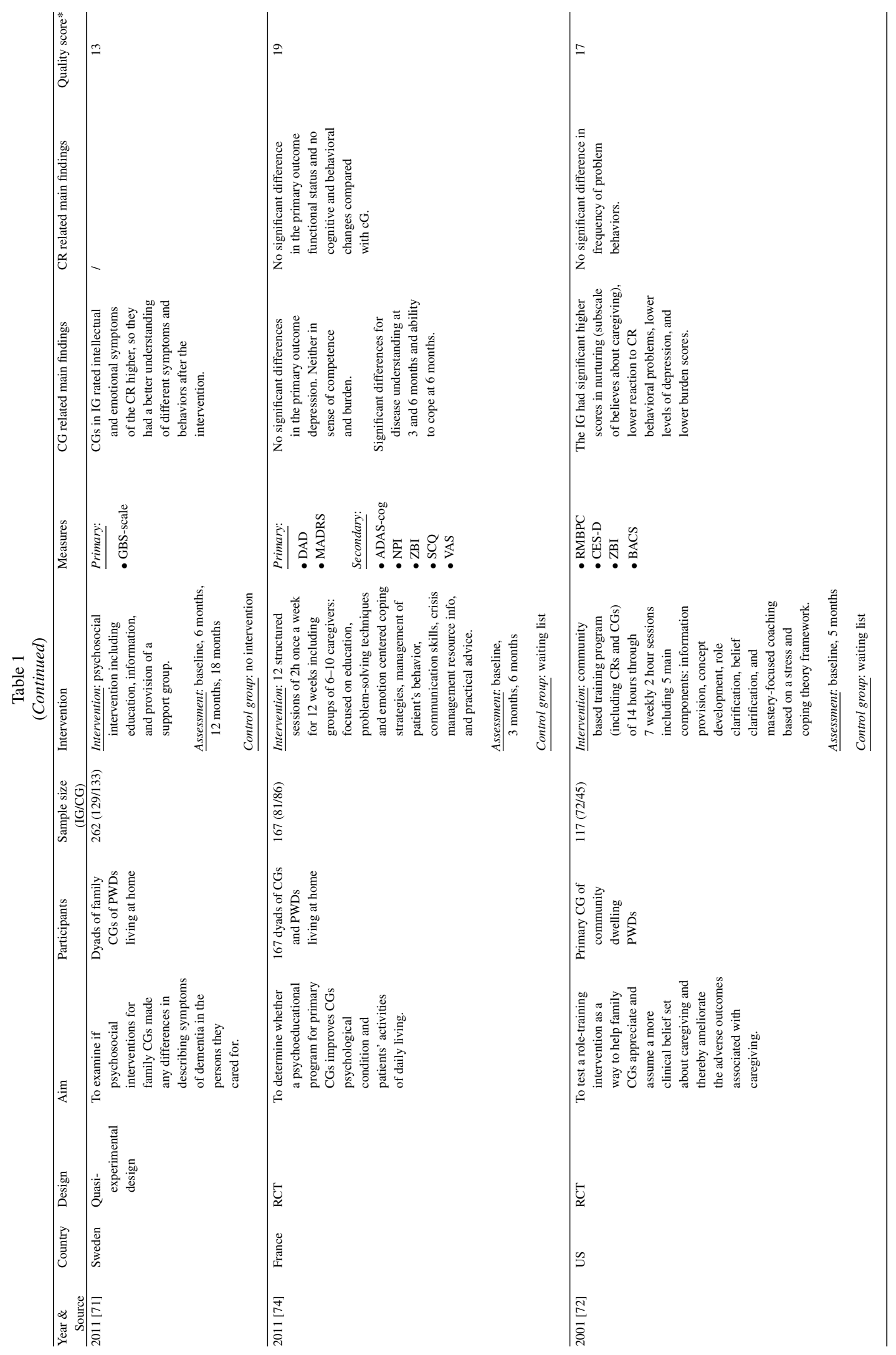




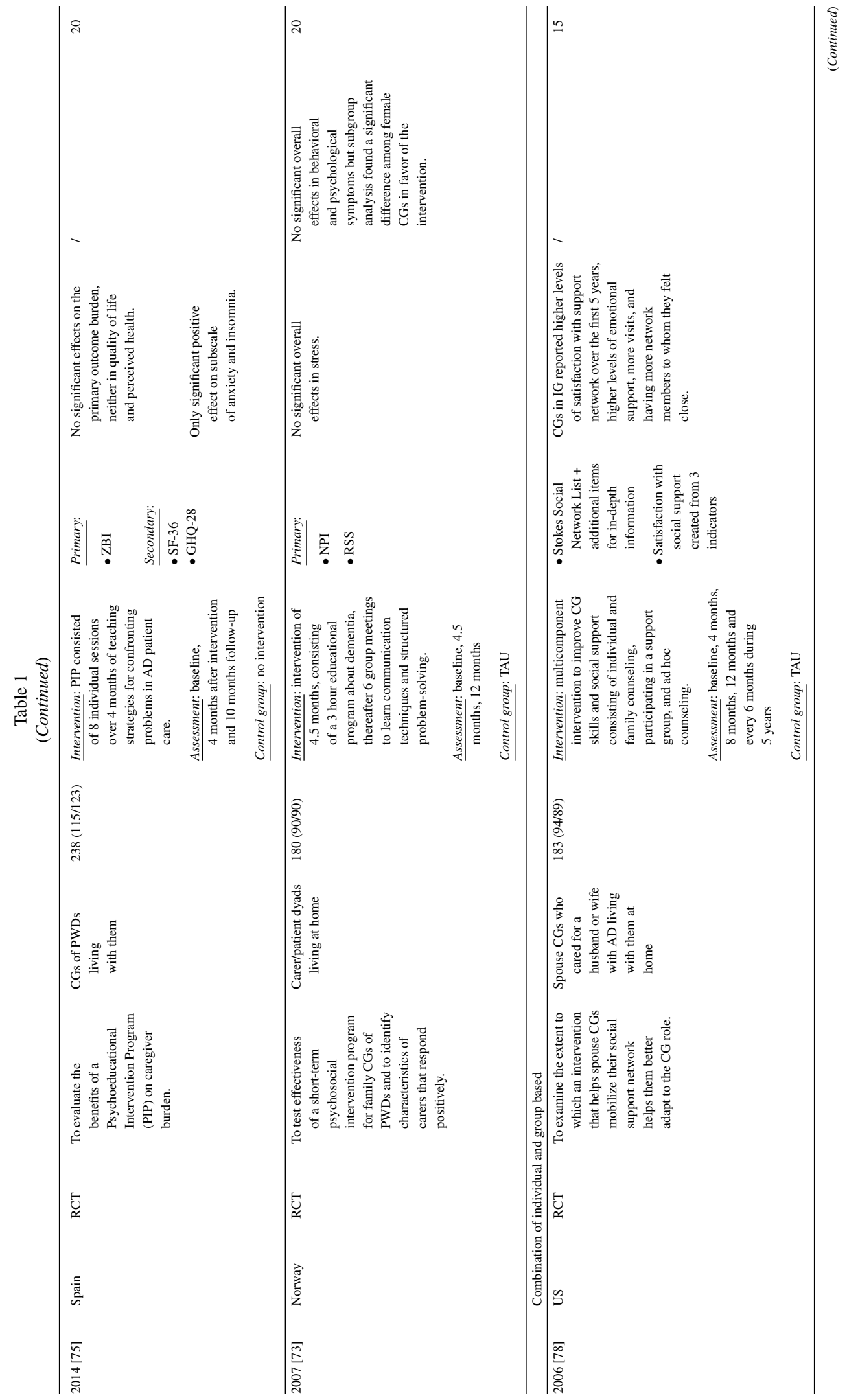




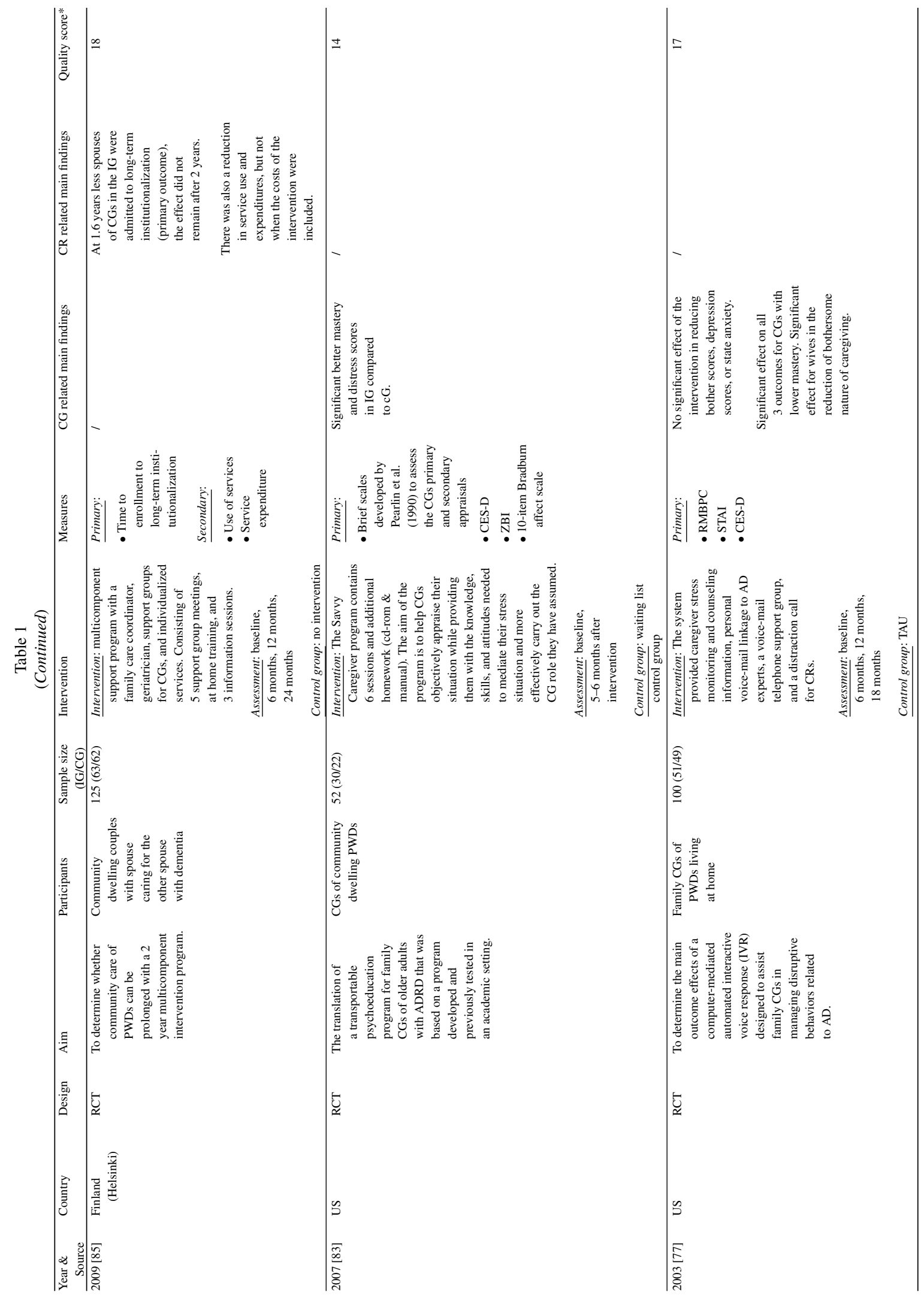




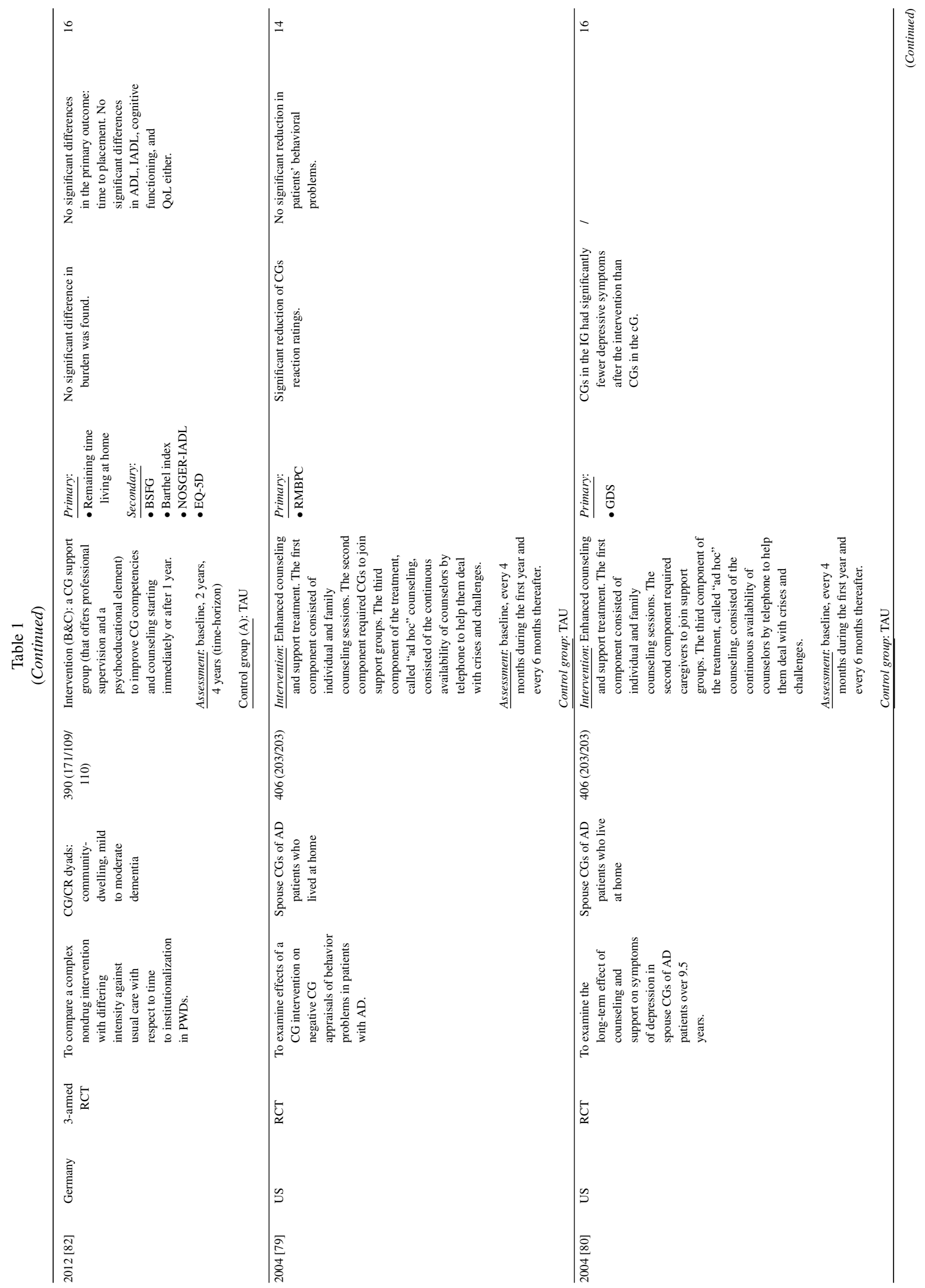




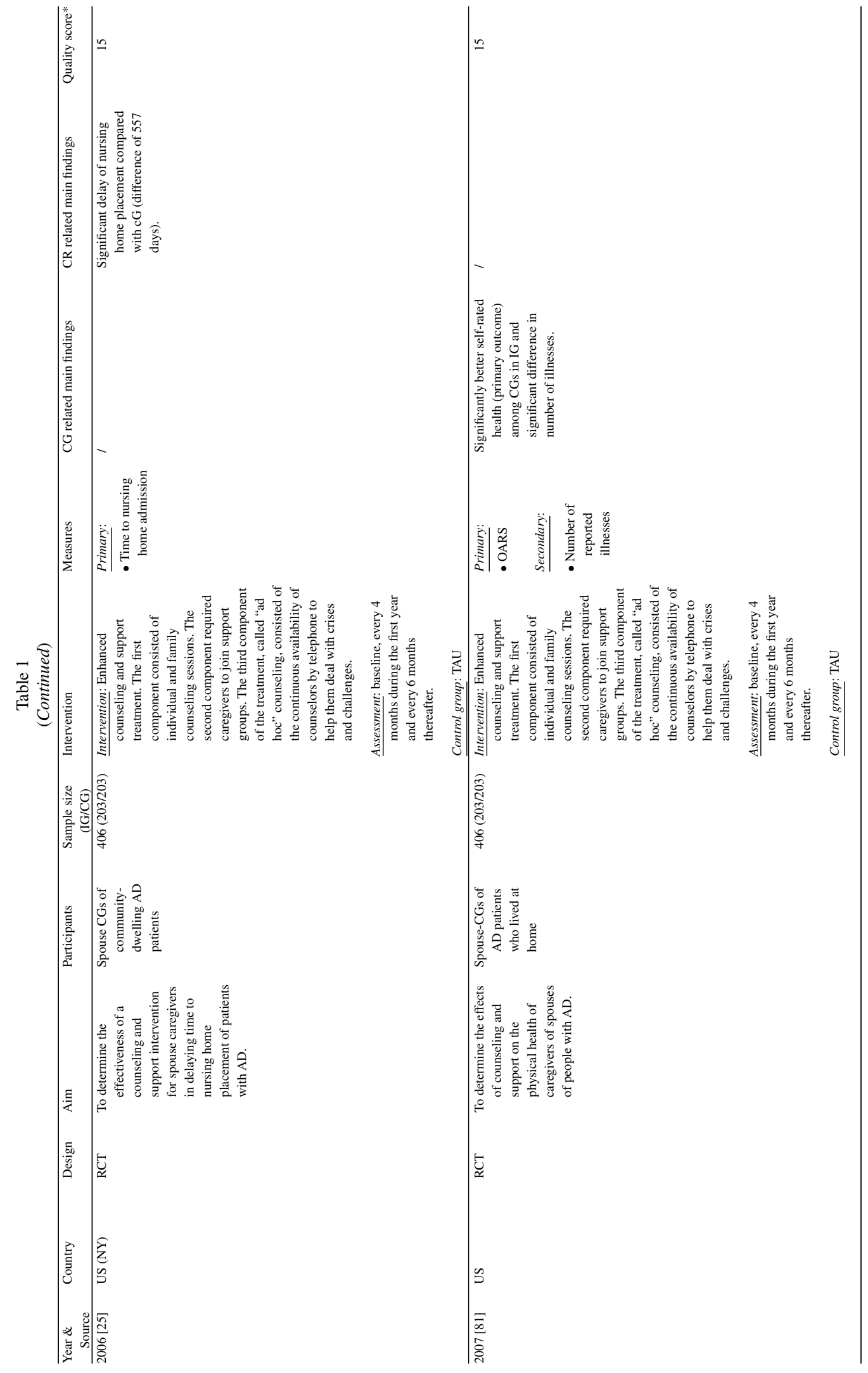




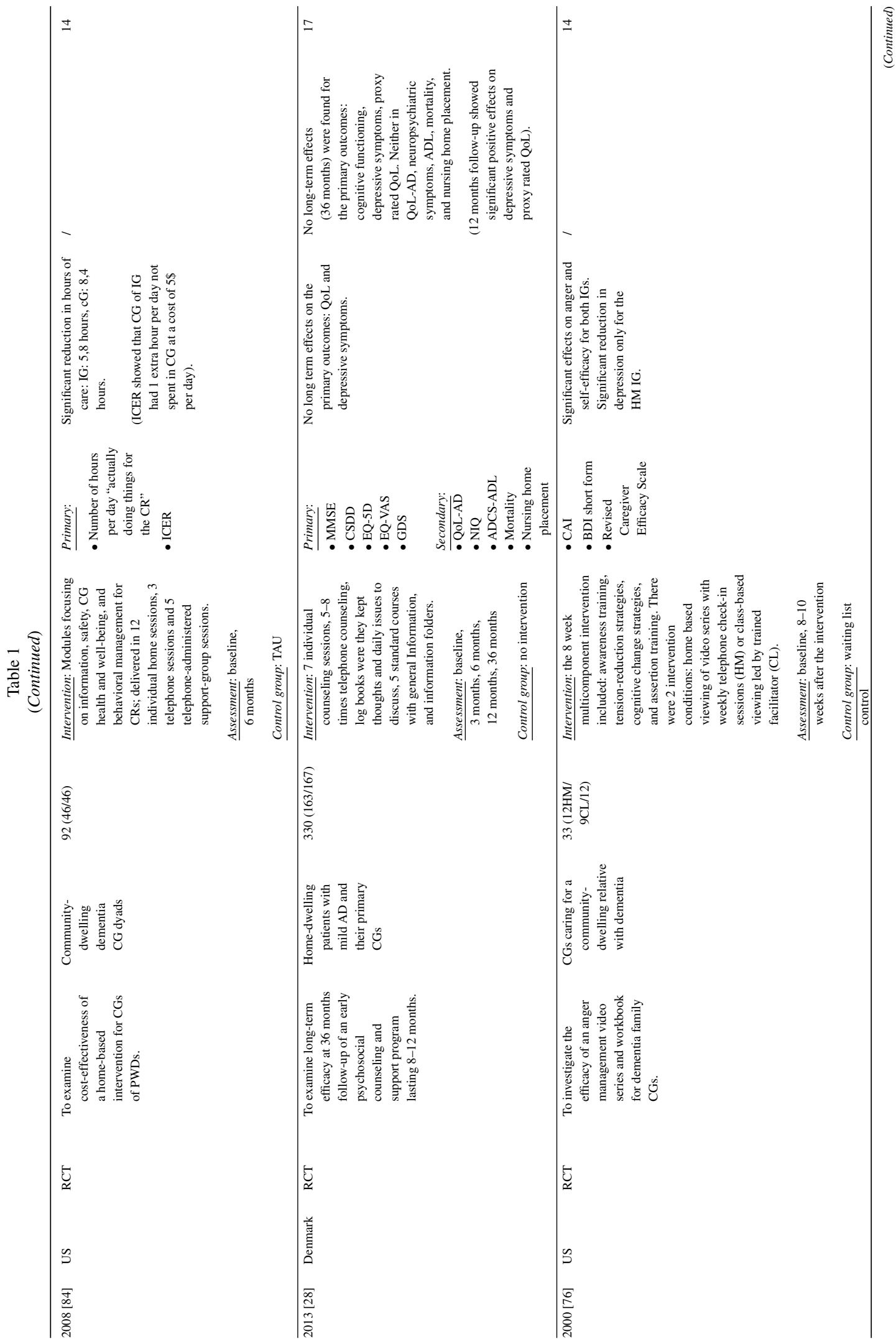




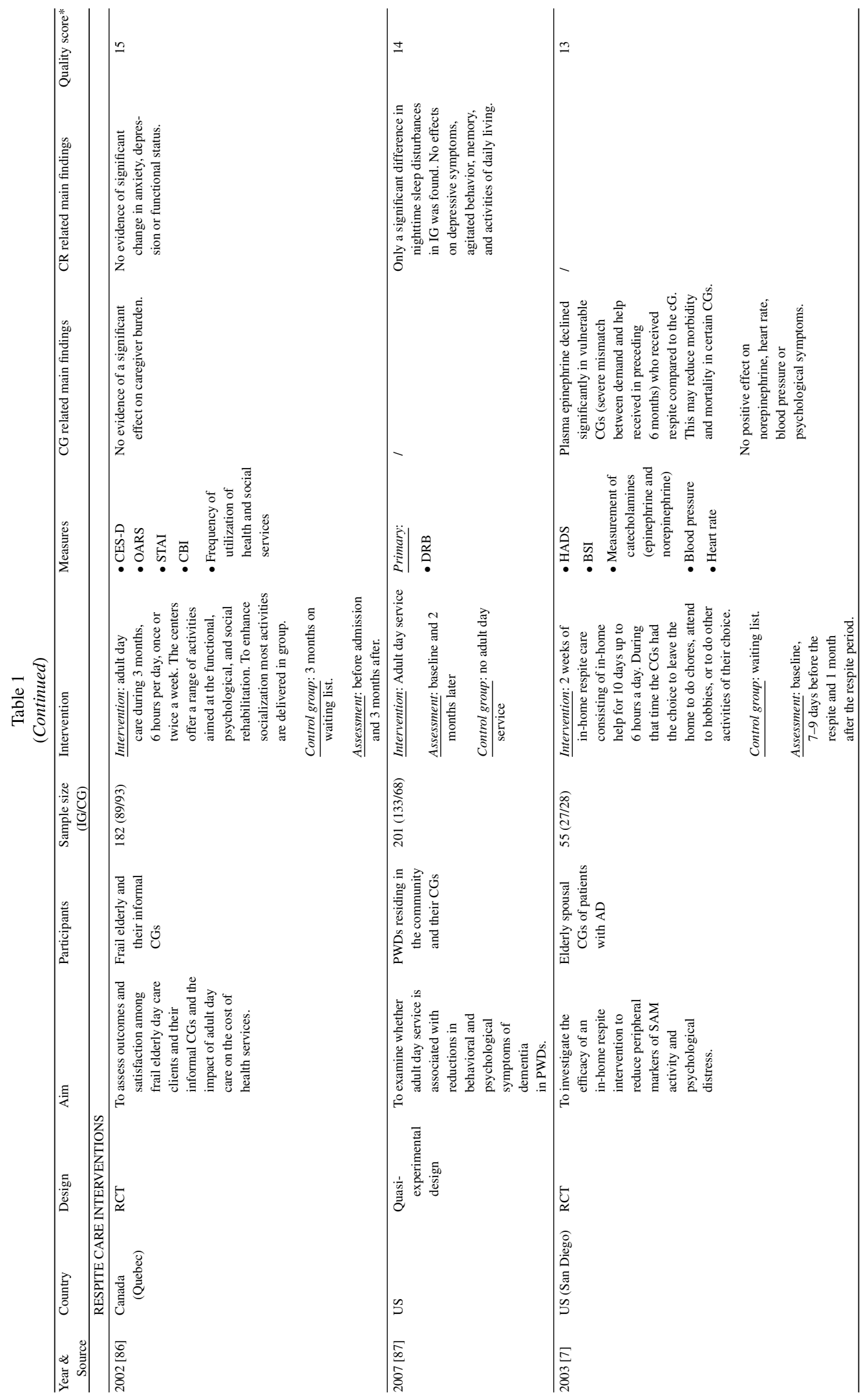




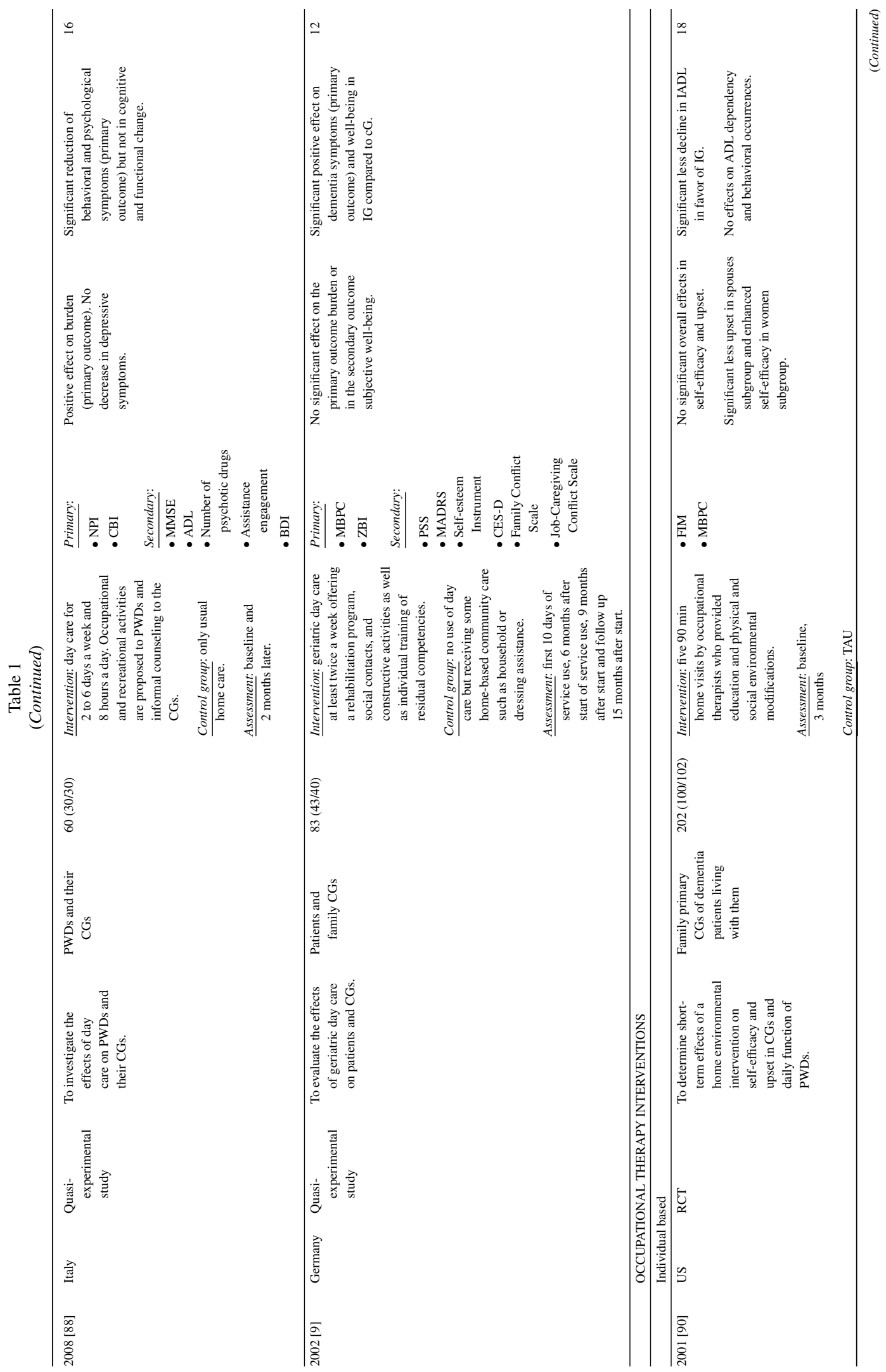



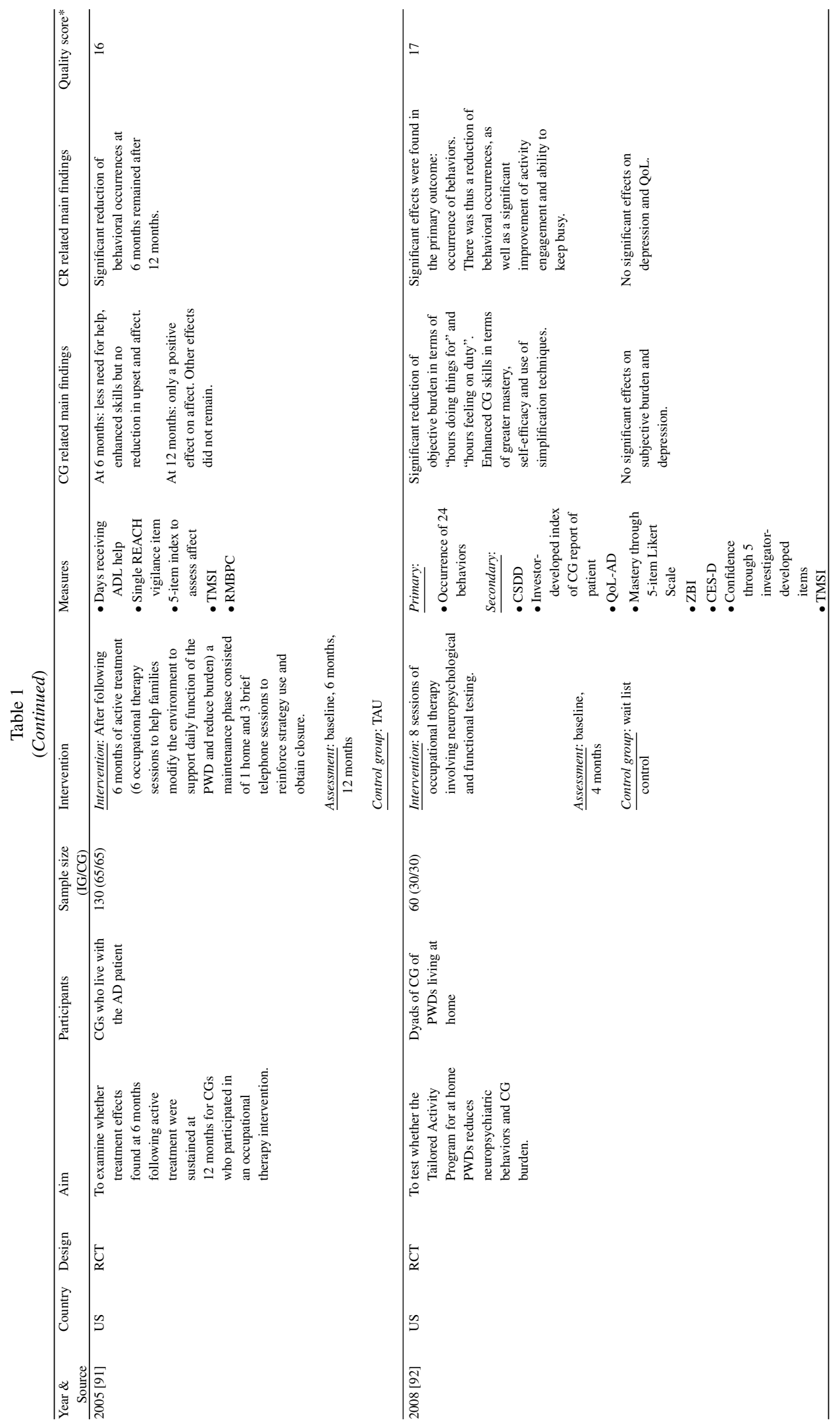


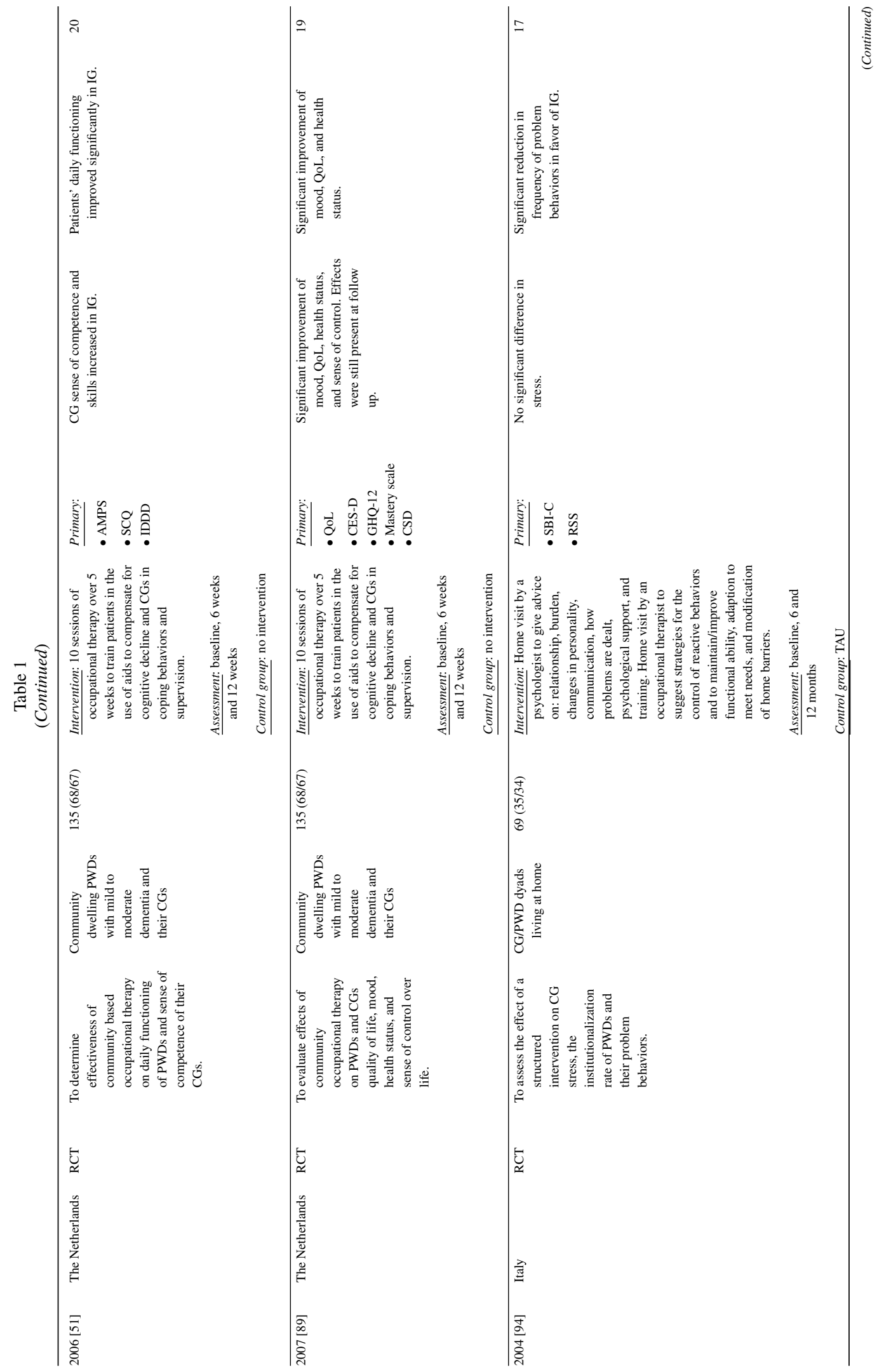




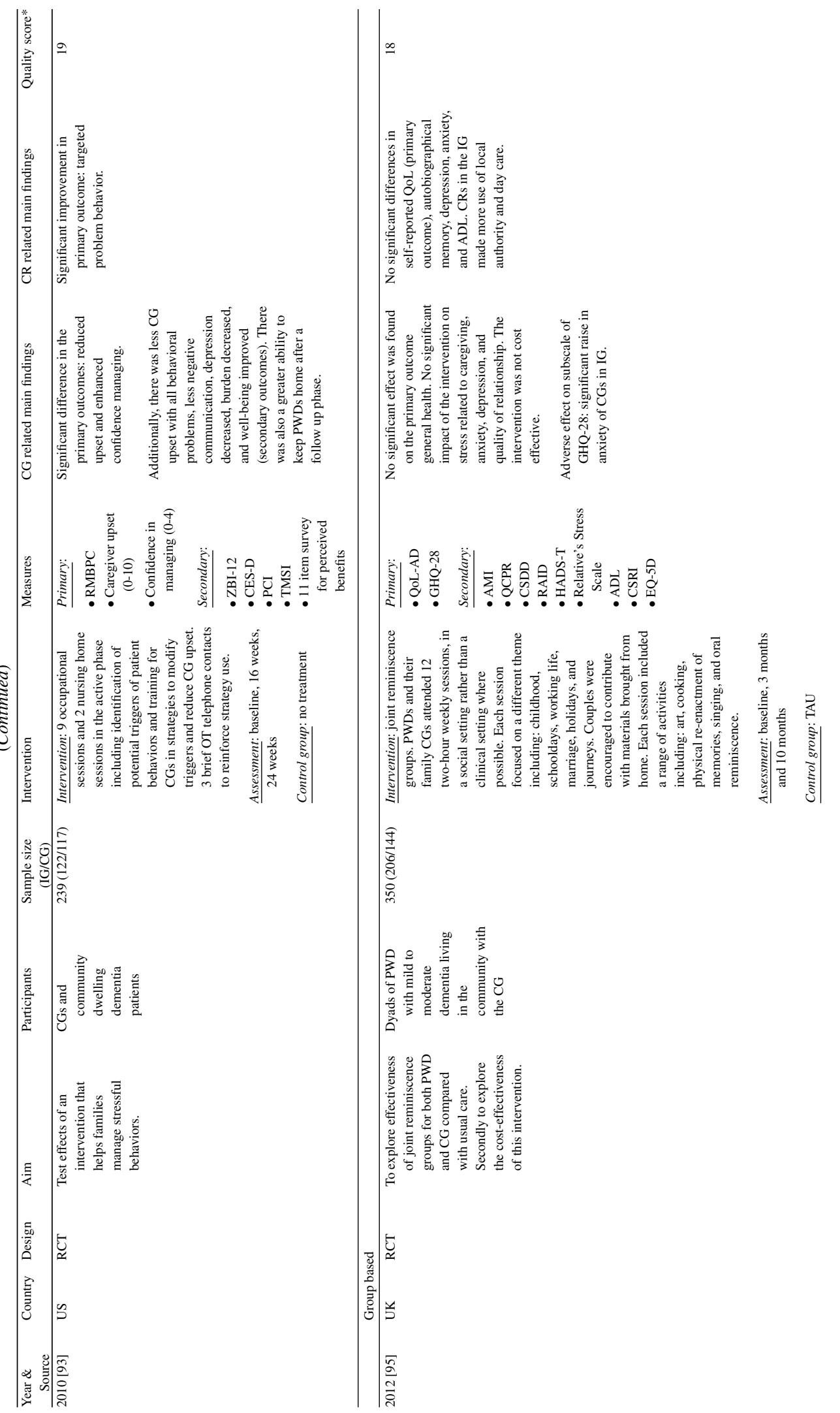




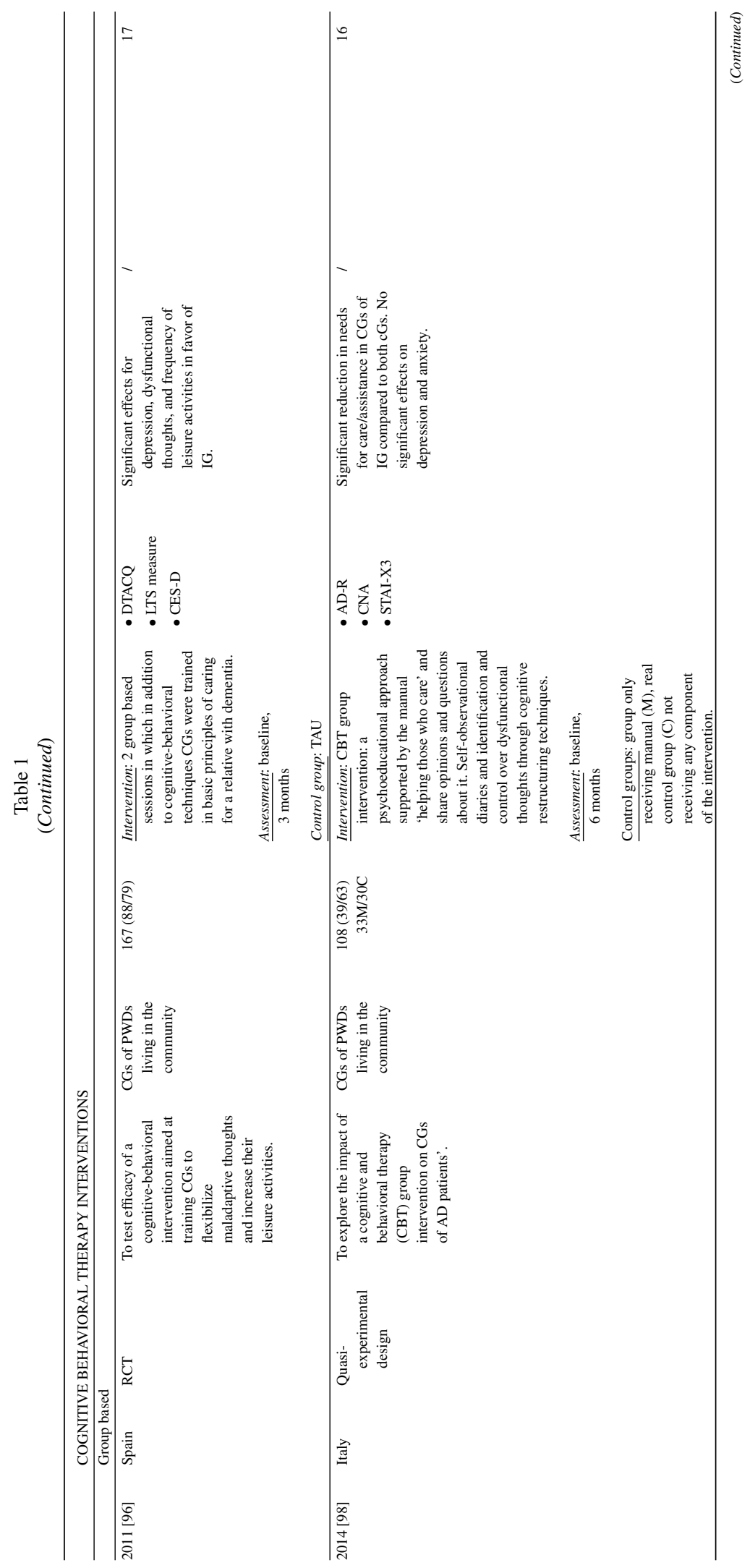




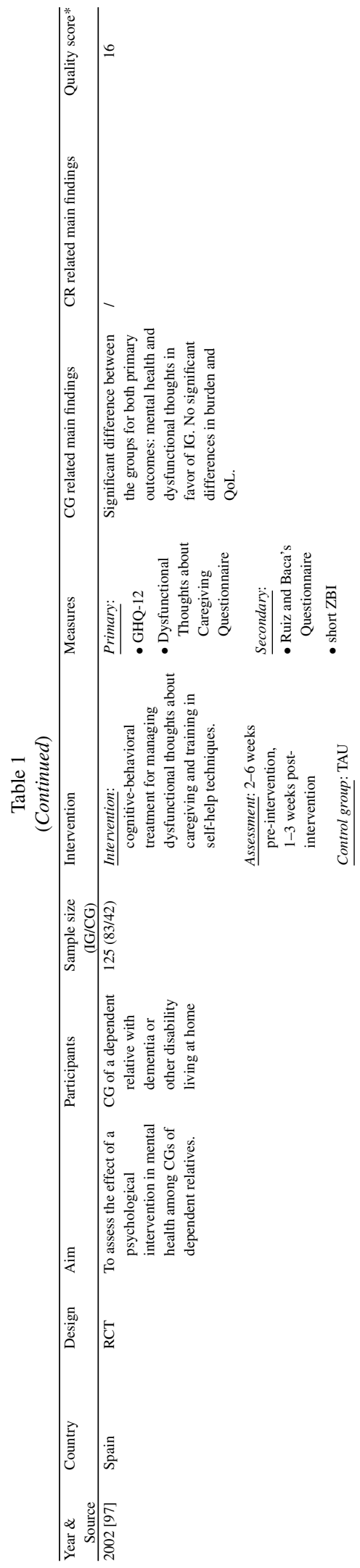

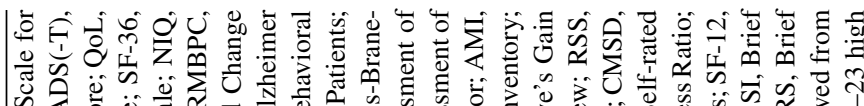

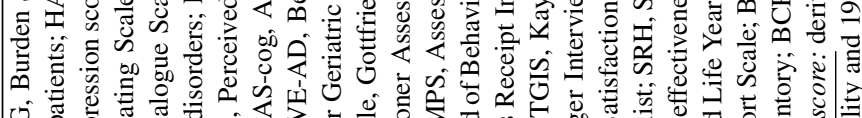
نे 空.

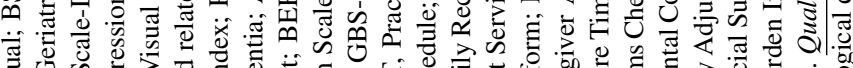

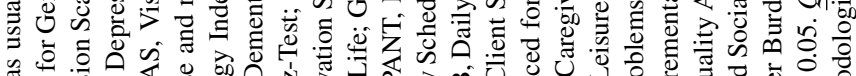

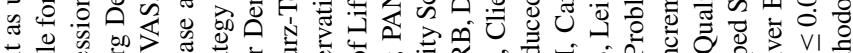

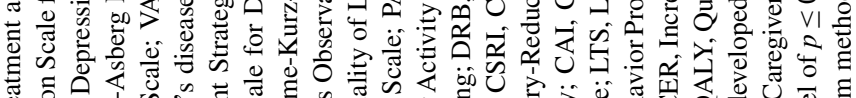

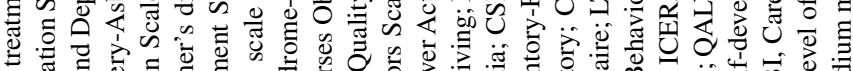

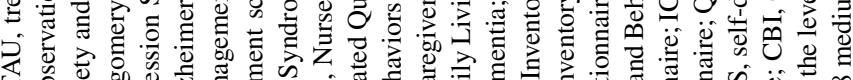

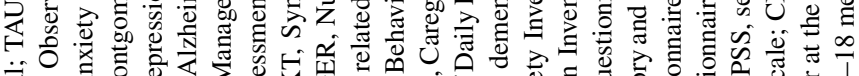

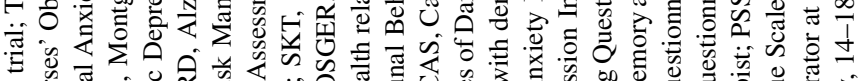

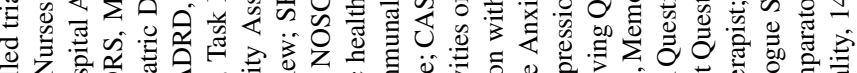

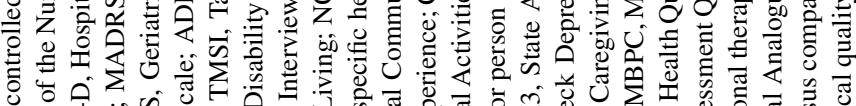

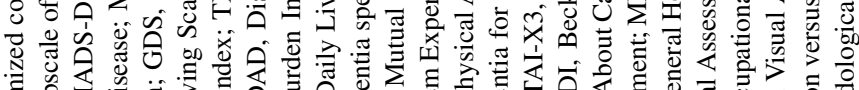

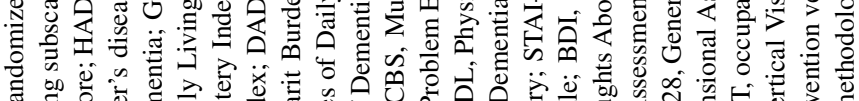

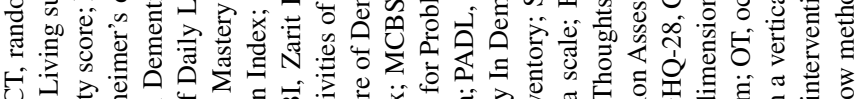

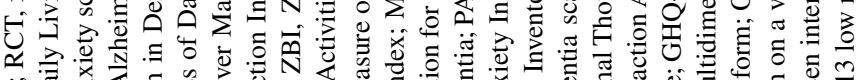

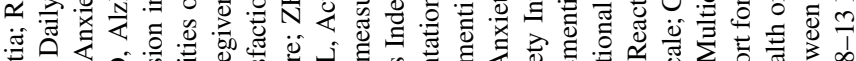

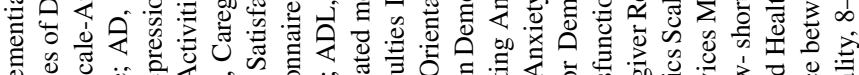

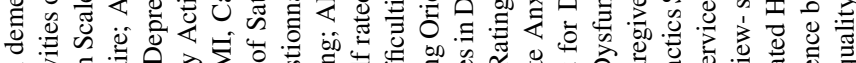

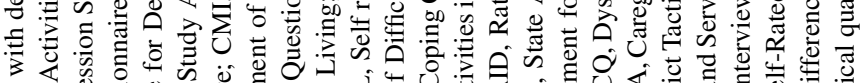

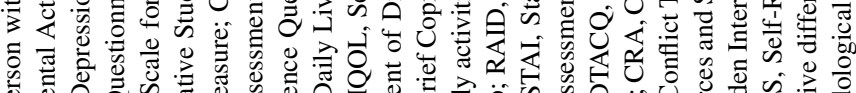

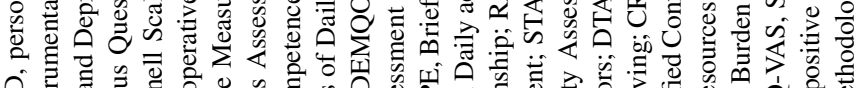

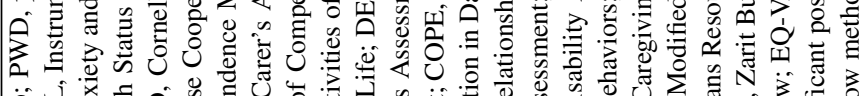

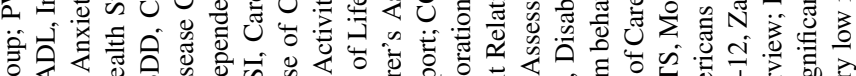

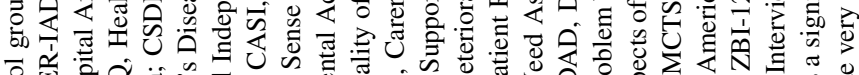

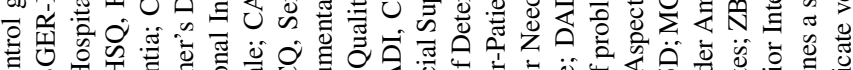

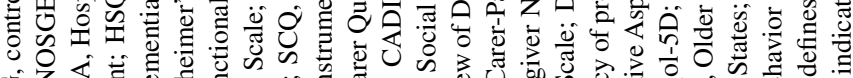

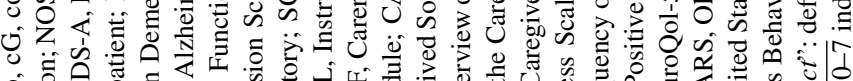

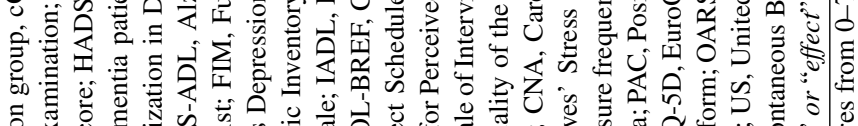

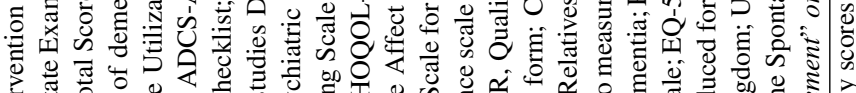

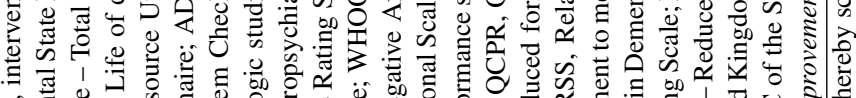

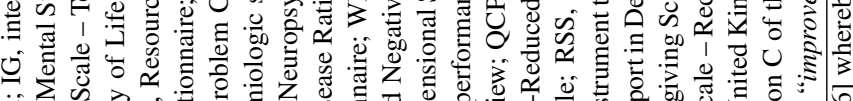

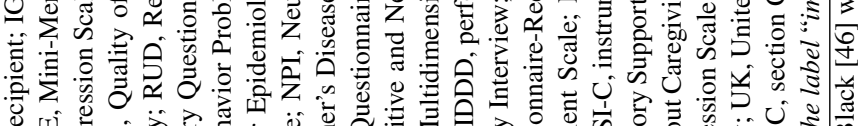

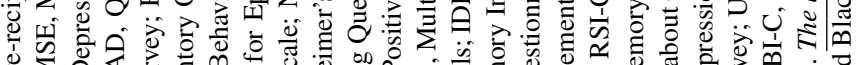

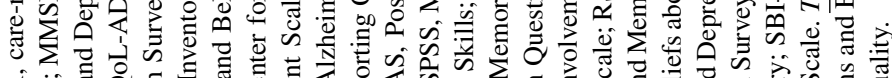

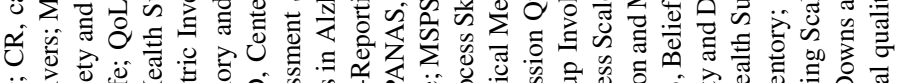

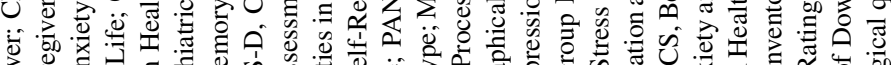

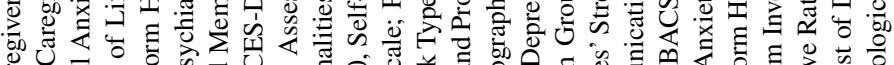

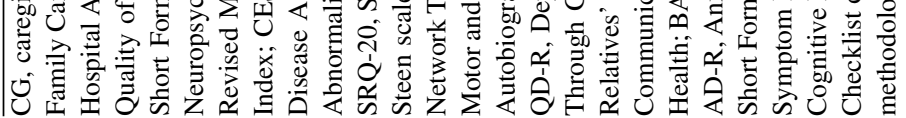


the caregivers. This was done by a care manager who helped caregivers create a care management manual [55]. Results indicated clear benefits for the intervention caregivers in the primary outcome adherence to guidelines and in multiple secondary resource and self-efficacy outcomes (receipt of services and resources, perceived quality of health care, confidence, mastery, social support, and receiving as much help as needed of formal caregivers). On the other hand, knowledge and quality of life of informal caregivers did not improve. Care-recipients also benefitted from the intervention. Their decline in quality of life was lower than in care-recipients of the other trial arm.

In another study of high methodological quality, informal caregivers received social and emotional support from a trained volunteer during home visits. This study could not produce any benefits in the primary outcome depression, nor in the secondary outcomes related to mental health, subjective well-being, resource use, and self-efficacy (anxiety, objective burden, resource use, QALY, life events, loneliness, affect, relationship quality, social support, and coping) [56].

Also Melis and colleagues sent a nurse to the homes of caregivers to support them. Although this intervention of medium methodological quality found no overall positive effects on the primary outcome caregiver burden and the secondary outcome time spent in caring, subgroup analysis showed benefits on burden of caregivers living with the patients [57].

\section{Group based single component interventions}

For the two group based single component psychoeducational interventions of medium methodological quality, only small benefits for caregivers were found. In one study, only a subgroup of caregivers benefitted, and in the other study, there was only some improvement found in a subscale of one of the outcomes under investigation.

The first intervention consisting of telephonebased support groups could not find benefits in the primary outcomes: depression, burden, and personal gains. However, a subgroup of older caregivers ( $>65$ years) reported lower depression rates in the intervention group [58].

The other study that implemented group-based education showed no overall positive effects on the primary outcomes: depression and quality of life of caregivers or in the secondary outcomes: resource utilization, hours spent in caregiving, and delay of temporary or permanent nursing home placement. Only emotional role functions improved (subscale of the health survey) [26].

\section{Combination of individual and group based single component interventions}

The only study, of high methodological quality, combining individual and group-based counseling could not find any improvements. No positive effects were found in the primary outcomes: depression and anxiety or in the secondary outcomes: burden and quality of life of intervention caregivers [59].

\section{Multicomponent interventions}

Twenty-eight of the 31 multicomponent interventions (eight of high, 20 of medium, and three of low methodological quality) showed improvements $(90 \%)$ on at least one of the investigated outcomes. Most frequently mentioned benefits for caregivers are a decrease in burden and depressive symptoms, and for care-recipients a delay in nursing home placement.

\section{Individual based multicomponent interventions}

Eleven studies were delivered individually and all showed improvements for the caregivers, especially in self-efficacy and burden. Five studies were evaluated as having high methodological quality, five as having medium methodological quality, and one as having low methodological quality.

Three different individual technology based interventions can be identified in this section. They all generated benefits for caregivers although results were rather mixed. A telephone-based intervention of medium methodological quality (containing emotional support, education, and teaching strategies) found reduced caregiver burden. In addition, caregivers also showed less severe reactions to memory and behavioral problems of the care-recipient. On the other hand this study could not identify a decrease in depressive symptoms of caregivers [60]. In contrast, a second study of medium methodological quality delivering a telephone-based exercise (consisting of a telephone-based exercise, a booklet, a workbook, and motivational newsletters) could not find a decrease in burden, but did find a decrease in perceived stress and exercise self-efficacy. On the other hand, efficacy in self-care and total weekly exercise did not improve, 
but caregivers with low exercise scores at baseline did show improved total weekly exercise [61]. A last technology based intervention of low methodological quality that provided two DVD based training sessions and communication support strategies could not demonstrate a reduction in burden either, but did have a positive impact on knowledge and positive aspects about caregiving. It also reduced the frequency of disruptive behaviors of care-recipients, but did not improve their mood [62].

Eight individually delivered interventions combined education and training. Each intervention that investigated the possible impact on caregivers indicated benefits for caregivers, especially in burden and self-efficacy outcomes. Teri et al., targeting their intervention of high methodological quality at both caregiver and recipient, through an exercise program for the recipients and training for caregivers, found positive effects on the primary outcomes physical health and depression (affective status) in recipients. Possible impact on the caregivers was not measured in this intervention [63]. An intervention of medium methodological quality, that added adaptive treatment to the education and problem-solving training, partially decreased subjective and objective burden and enhanced caregivers in terms of upset. There was also less need for ADL help and better affect. On the other hand there were no differences found for caregivers' overall well-being and overall (objective and subjective) burden, nor in care-recipients' behavioral problems and in their physical functioning [22]. The psychoeducational intervention program (PIP) of high methodological quality developed by Martin-Carrasco et al. also decreased burden (primary outcome), improved mental health, and did improve caregiver well-being [64]. In accordance, Gavrilova and colleagues, who also implemented a high quality multicomponent intervention containing education and training, pointed out a decrease in caregiver burden, but could not decrease distress or improve quality of life. For recipients, no differences were found in quality of life, nor in behavioral and psychological symptoms [23]. Ducharme et al. concluded that after receiving their intervention of medium methodological quality, also containing education and training, caregivers were more confident in dealing with caregiving situations. They perceived themselves better prepared for caring, more efficacious in their role, and were better able to plan for future needs. They also had a better knowledge of services and made more frequent use of coping strategies. No significant differences were found for perceived informal support and family conflicts [65]. In Ducharme et al. (medium methodological quality), the authors gave half of the intervention caregivers a booster session wherein the caregivers were asked about changes in the caregiving situation over the last 6 months. Only the preparedness to provide care differed significantly between the caregivers receiving the booster session and those who did not [66]. Finally, another intervention of high methodological quality implemented a coping based psychoeducational intervention with educational and training components. Intervention caregivers had reduced affective symptoms, i.e., depression and anxiety (primary outcome), reduced case level depression, and improved quality of life. On the other hand case level anxiety and potential abusive behavior of caregivers, and quality of life of recipients [24] did not improve. The intervention remained effective after 24 months and was also cost effective [67].

\section{Group based multicomponent interventions}

Seven of eight multicomponent studies (three of high, three of medium, and two of low methodological quality), delivered to groups, indicated positive effects. Six of them found benefits for caregivers and two for care-recipients. Although most interventions benefitted caregivers, the found effects were rather small and often only found in subscales of the measurements.

An intervention of low methodological quality containing elements of education, support, and skills training could not find overall effects on burden or satisfaction of caregivers. Only strain, disappointment (subscales of the used burden scale), and purpose (subscale of the used satisfaction scale) showed improvements [68]. The same authors also investigated possible impact of the intervention on time to nursing home placement but could not find significant delay, except for a subgroup of caregivers of adult children and daughters [69].

Berger et al. implemented a dyad intervention (medium methodological quality) by combining a support group for caregivers with music therapy for recipients. Burden and depressive symptoms of caregivers did not decrease. Neither did behavioral and psychological symptoms, and cognitive and functional status of recipients after receiving the intervention [70]. Another quasi-experimental intervention of low methodological quality also providing a support group, but this time in combination with education and information sessions, increased 
caregivers' disease understanding (primary outcome) [71].

Four different studies described interventions with training and educational components to caregivers. One of them (medium methodological quality) found lower levels of depression and burden of caregivers beside less severe reactions to behavioral problems. There was, however, no decrease in the frequency of problem behaviors of recipients [72]. In contrast, an educational program of high methodological quality followed by group meetings to learn communication techniques and structured problem solving, did decrease behavioral and psychological symptoms (primary outcome) of recipients in a subgroup of female intervention caregivers. Caregiver stress (primary outcome) on the other hand was not reduced [73]. Another intervention of high methodological quality found significant improvements in disease understanding. Even more, the intervention increased caregiver ability to cope, but could not decrease depressive symptoms (primary outcome), sense of competence, and burden of caregivers. Neither could the intervention induce functional (primary outcome), cognitive, and behavioral changes in recipients [74]. The intervention of high methodological quality developed by Martin-Carrasco et al. was more recently also implemented in groups. This time, the authors could not establish the same benefits as in the individual delivered psychoeducational intervention. Burden (primary outcome), quality of life, and perceived health did not improve in caregivers. Only some benefits were identified on a subscale of anxiety and insomnia [75].

\section{Combination of individual and group based multicomponent interventions}

Twelve psychoeducational interventions, of medium methodological quality, that partially delivered their intervention in group and partially on an individual basis were included in this review. Ten of them benefitted caregivers or care-recipients. Most frequently reported benefits were in self-efficacy and depressive symptoms of caregivers, and in delay of nursing home placement of care-recipients.

Two technology-based interventions can be classified in this section. Steffen, who investigated the effectiveness of an anger management video series, containing multiple training strategies, on caregivers found significant positive effects on anger, self-efficacy in managing problem behaviors, and also on depressive symptoms in the home based viewing group [76]. On the other hand, another computer-mediated automated interactive voice system intervention-containing stress monitoring, information, counseling, a telephone support group, and a distraction call for recipients-only showed positive effects in reducing bother, depressive symptoms, and anxiety in caregivers with lower mastery. For the subgroup of wives, there was a reduction in bother [77].

Drentea and colleagues combined individual and family counseling with participation in a support group. They found higher levels of satisfaction with their support network over the first five years, higher levels of perceived emotional support, more visits, and having more network members to whom they felt close in the intervention group [78]. Another similar intervention composed of counseling and support groups found a significant reduction in caregiver reactions to behavioral problems (primary outcome), but not in patient behavioral problems [79]. They also found positive effects on depressive symptoms of caregivers (primary outcome) [80] and also pointed out that recipients of the intervention group stayed on average 557 days longer at home than those in the control group (primary outcome) [25]. Finally, they concluded that intervention caregivers had significantly better self-rated health (primary outcome) and that their number of reported illnesses decreased [81]. In contrast, other authors [82] who also combined a support group with counseling could not find to same benefits on time to placement (primary outcome). Even more, caregiver burden, and care-recipient's ADL, IADL, cognitive functioning, and quality of life in the intervention groups did not change.

The Savvy Caregiver program, that implemented an intervention containing educational and skills training components to support caregivers, concluded that interventions caregivers had better mastery and distress scores compared to those who did not receive the intervention [83].

Nichols et al. conducted an intervention combining education with support group provision. They found significant reduction in hours of care (primary outcome) provided by the caregiver and concluded that the intervention was also cost-effective [84].

Eloniemi-Sulkava et al. implemented an intervention containing support group meetings, at home training, and information sessions. Less recipients of the intervention group were admitted to longterm institutionalization after 1.6 years (primary outcome), but the effect did not remain after 2 years. 
The study also found a reduction in service use and expenditures. However, the reduction diminished when costs of the intervention were included [85].

Phung and colleagues investigated long term effects of an intervention combining counseling and education that already showed benefits on the primary outcomes depressive symptoms and quality of life of recipients at 12 months. After 36 months the earlier found benefits did not remain. The other investigated outcomes did not improve or decrease [28].

\section{Respite care}

Five respite care interventions, two of low methodological quality and three of medium methodological quality, were included. Four measured the impact of day care and one of an in-home respite care program. Although three of the four day care interventions indicated benefits for the care-recipient (75\%), especially in decreasing behavioral problems, the reported benefits of day care provision for caregivers were rather small and mixed (33\% were effective). The only included in-home respite care program indicated some beneficial effects for caregivers, but did not investigate the impact on the care-recipient.

An intervention of medium methodological quality providing three months of day care once or twice a week could not find an effect on caregiver burden or any benefits for the care-recipients in anxiety levels, depressive symptoms, or functional status [86]. On the other hand another intervention of low methodological quality providing day care at least twice a week did report a decrease in dementia symptoms in care-recipients and increased their well-being. Also in this intervention, there was no effect on caregiver burden or in subjective well-being [9]. These findings partially corresponds with the findings of Femia et al. (medium methodological quality) who also reported an improvement in nighttime sleep disturbances, a subscale of total behavioral problems (primary outcome) [87].

In contrast to the others, Mossello et al. did find a decrease in caregiver burden (primary outcome) besides a decrease in behavioral problems of carerecipients (primary outcome). It must however be mentioned that in this medium quality intervention the caregivers also received some additional counseling in the day care center [88].

The only in-home respite care program (low methodological quality) that was included provided two weeks of in-home help during six hours a day. Some beneficial effects for caregivers were found which can reduce their morbidity and mortality [7]. On the other hand benefits in psychological symptoms could not be found.

\section{Occupational therapy interventions}

Eight occupational therapy interventions (three of high methodological quality and five of medium methodological quality) were included. All investigated the impact on the well-being of the caregiver and on the care-recipient. All seven occupational interventions delivered to individuals showed benefits, especially in caregiver self-efficacy outcomes and in the frequency of behavioral problems of care-recipients. The only group based occupational therapy intervention could not find benefits.

\section{Individual based occupational therapy interventions}

Graff and colleagues developed a community based occupational therapy intervention of high methodological quality containing ten sessions to train both caregivers and care-recipients. Both caregivers and care-recipients benefitted from the intervention. In caregivers of the intervention group sense of competence, skills, and sense of control improved. For both caregivers and recipients mood, quality of life, and health status improved. In carerecipients daily functioning improved [51, 89].

Another research team implemented three similar occupational therapy interventions of medium methodological quality, each time in a different dose of intensity. For all three different doses, benefits were found for the dyads under investigation. During the first intervention, participants received five occupational therapy sessions. Here, only subgroups of caregivers (spouses and women) benefitted. They indicated less upset and enhanced self-efficacy. Recipients showed less decline in IADL, but not in ADL or in behavioral problems [90]. In the second intervention, they provided six sessions of occupational therapy. This time behavioral problems of the recipients did decrease. For all caregivers, reported need for help and skills improved, but no decline was found in caregiver upset. There were also no changes in affect at 6 months. Nevertheless, over a longer period affect improved [91]. In the last intervention, dyads received eight sessions. Here, the same improvements as in the previous intervention were found for behavioral problems and skills. Even more, recipients showed improved activity engage- 
ment and ability to keep busy. Caregivers showed a reduction in objective burden. On the other hand no effects were found on depressive symptoms of the dyads, on subjective burden of caregivers, and on quality of life of recipients [92]. Yet another occupational therapy intervention (high methodological quality) of the same first author as the previous studies [93], that contained nine occupational therapy sessions, showed overall positive effects on the primary caregivers' outcomes: upset and confidence in managing target problem behavior as well as in the care-recipient primary outcome: frequency of target problematic behavior. Additionally, there was less caregiver upset with all problem behaviors, less negative communication, depression and burden decreased, and well-being improved (secondary outcomes). Finally, after the follow up phase intervention caregivers had a greater ability to keep patients home.

Finally, Nobili and colleagues added an educational component to their occupational therapy intervention of medium methodological quality. Although the intervention could not reduce stress in caregivers, the frequency of behavioral occurrences of recipients also decreased after implementation of their intervention [94].

\section{Group based occupational therapy interventions}

Unlike the other individual occupational therapy interventions, the only occupational therapy intervention in group (medium methodological quality) did not show any benefits for the dyads [95]. None of the investigated caregiver outcomes improved (primary outcome: general health of caregivers \& secondary outcomes: distress, depression, relationship with the recipient, and anxiety). Neither did the investigated care-recipient outcomes (primary outcome: quality of life \& secondary outcomes: autobiographical memory, depression, anxiety, ADL, and service use). Even more, a side effect was identified. Caregivers of the intervention group had higher anxiety levels on a subscale (GHQ-28) after implementation.

\section{Cognitive behavioral therapy interventions}

Three group-based cognitive behavioral therapy interventions, each of medium methodological quality, delivered in groups were included. In each study benefits for caregivers were found especially in dysfunctional thoughts. Possible impact on the carerecipient was not measured in any of the studies.
The first group of authors who tested efficacy of a cognitive behavioral therapy intervention found positive effects on dysfunctional thoughts, frequency of leisure time, and depressive symptoms [96]. The second group of authors who added training in selfhelp techniques to the cognitive behavioral treatment also found a positive impact on the primary outcomes: dysfunctional thoughts and mental health of caregivers of the intervention group. The secondary outcomes quality of life and burden did not differ after receiving treatment [97]. Finally, Passoni et al. concluded that both intervention groups had less need for care or assistance compared to control group participants, but anxiety and depressive symptoms did not differ [98].

\section{DISCUSSION}

A first major strength of this paper is its general overview and update in the knowledge of supporting strategies targeting informal caregivers of people with dementia by searching for potential new insights based on recent interventions, and by balancing previous knowledge against current findings. Additionally, we explored whether the earlier identified methodological issues were still present. Finally, we attempted to develop a new classification to distinguish global and more specific tendencies in the results. As a result, we were able to state for each distinct supportive type what particular benefits are to be expected. After all, before one proceeds to invest in caregiver support, one must know what benefits to expect. Nevertheless, we have to recognize that the 53 studies describing interventions to support caregivers were multifaceted making it impossible to prevent that some interventions placed in one subdivision did not contain elements of others.

Another general limitation that should be mentioned is the lack of performing a quantitative analysis. This procedure was considered, but was not feasible due to methodological heterogeneity, i.e., variations in the outcomes and the various instruments used to measure these outcomes. Also, due to restricted resources available, the quality appraisal could not be fully evaluated by two independent authors. Nevertheless, a more pragmatic approach was used maintaining the methodological quality of the work at the highest possible level, i.e., it was opted to perform a quality appraisal of randomly selected studies by two reviewers independently, and then to compare their scores. Since the authors had 
very similar ratings and there was complete agreement on the level of quality the rest of the quality appraisal was done by one author following the same consistent approach.

In general most supportive interventions were to some extent effective $(87 \%$ of the studies showed at least some benefits for the caregiver and/or the care-recipient). This, however, should be interpreted with caution and not be overestimated. Indeed, effects were found in $87 \%$ of the included studies, but in some cases the benefits could only be found in secondary outcomes or even in subscales of outcome measures or in subgroups of participants. Even more, not all studies clearly specified their primary and secondary outcomes. It should also be mentioned that methods and findings were rather inconsistent. This phenomenon, also previously described by others, e.g., $[11,12,30,33,99]$, can still be explained by the heterogeneous nature of supportive strategies caused by differences in content, duration, and intensity; the methodological quality of studies; the variety in outcome measures; and the different characteristics of the caregivers and the care-recipients.

Another general conclusion that can be drawn is that individual interventions are more likely to be effective. Given the fact that caregivers are a heterogeneous group with different needs, expectations, and characteristics, it is to be expected that interventions delivered at individual level can better adapt to those differences and as a result lead to better results. This conclusion corresponds with the systematic review of Selwood. This author, who investigated the effect of psychological interventions on family caregivers of people with dementia, also concluded that group interventions are less effective [45].

\section{Methodological issues}

Based on our performed quality appraisal using the Downs and Black checklist [46], we identified 14 studies of high methodological quality, 34 of medium methodological quality, and five of low methodological quality. All high quality studies were effective except for two single component psychoeducational interventions of which we know they generally are less effective than multicomponent interventions. Even more, we have established that in nine of the 12 effective high quality studies, overall effects were found in the primary outcomes. On the other hand, 29 of the 34 studies of medium methodological quality were to some extent effective, but here only 20 studies could ascribe these benefits to significant changes in primary outcomes. Finally, all five low quality interventions were to some extent effective. However, only two of those studies found clear effects in the primary outcomes targeting caregivers.

Despite several previous reviews concluded that the interventions under study are overall effective in decreasing or increasing specific outcomes that improve well-being (e.g., depression, self-efficacy), our opinion is that these overall conclusions should be made cautiously due to inconclusive results often caused by methodological issues as has been discussed in other comparable reviews, e.g., $[11,12,30$, $33,37]$.

As long as researchers keep using a wide range of instruments to measure effectiveness, differences in effectiveness results could rather be caused by differences in sensitivity and specificity of the used measurements rather than by the fact that a certain intervention is less or more effective in influencing a certain outcome. Hence a standardized way of measuring outcomes is preferred. The latter might be perceived as a paradox since a person-centered approach is to be preferred. Yet, a compromise can be found by applying person-centered care while using standardized yet sensitive instruments to measure outcomes, such as the Zarit Burden Interview for caregiver burden.

The current interventions in this research area are also plagued by other methodological issues. First, the active components of the implemented interventions are often not enough described in detail making it difficult to attribute significant improvements to a particular type of intervention. Second, most interventions have short follow-up periods. Given the fact that dementia is a slow degenerating process with high demands for caregivers over long periods, knowledge on long-term effects can be important as well. Yet another frequently occurring shortcoming is the absence of a well-performed power calculation to determine the appropriate sample size. This often resulted in too small sample sizes. As a result, a risk of falsely rejecting effective interventions arises because the sample was not large enough to establish effects. Simultaneously occurring with this issue is the lack of exploring the minimal needed effect size of the research outcomes.

Knowing the characteristics of participants lost to follow up and their potential significant differences with those who completed the study can provide researchers important information about the acceptability and applicability of interventions. Nevertheless, in several studies this important step was 
not accomplished. Also, studies often lacked to ascertain that subjects asked and prepared to participate were representative of the entire source population.

Another more difficult methodological problem to solve is about the control group. In the included studies, the control group is mostly defined as the group not receiving the intervention or only receiving treatment as usual. Because it is often not clear what other supportive services they get besides the intervention, benefits could also be caused by those other services. Even more, subjects that are potentially most in need of support could be forgotten because most dyads are recruited via organizations already giving them some support. In the future, researchers should explore more how to find those subjects. Also including them can contribute to finding stronger evidence.

Finally, dementia can be divided into different stages. Although some interventions mention in what stages the included subjects were, it is not known what influence severity of dementia can have on certain effective interventions.

\section{Different types of interventions}

Psychoeducational interventions are the most commonly investigated type and in general an effective strategy ( $86 \%$ of the studies showed benefits) also showing the typical inconsistency that we already discussed. Of this broad type, the multicomponent interventions are most popular. Many multicomponent studies reported a positive impact on caregivers (83\%) especially in self-efficacy, burden, and depressive symptoms. However, they had in general less effect on care-recipient outcomes (39\%) except for delay of nursing home placement. As already mentioned, the single component psychoeducational interventions solely focusing on one strategy (such as education, social support) were rather not effective. This does not mean that these strategies should not be undertaken, but rather that they need to be embedded into more extensive interventions.

Although not many cognitive behavioral therapy interventions were included in this review, this supportive strategy seems to be effective in decreasing dysfunctional thoughts of caregivers. Future research of this type should, however, consider measuring the possible indirect effects on care-recipients as well.

Occupational therapy interventions also seemed to be effective in decreasing behavioral occurrences of care-recipients and self-efficacy of caregivers, especially when there were delivered individually.
Only a few studies about some types of respite care were included in this review. Four of the included studies provided day care allowing the caregiver to take a break. Unfortunately, only one community based in-home respite care intervention was included. Knowing that substantial research, especially qualitative research, was already done in this field there seems to be a lack of RCTs and quasi-experimental designs measuring the impact of certain respite care services [38]. This could be explained by the difficulty of finding a similar control group, leading researchers to choose a pre-posttest design. Although most included day care interventions indicated benefits for the care-recipients in terms of behavioral problems, caregivers outcomes were rather mixed. In accordance to the main findings of the recently updated review of Lee et al. [100] by Maayan et al. [37], this review also concludes that the mixed results and the small number of included studies in this area make it impossible to draw clear conclusions about effectiveness of respite care services. Therefore, an updated review about effectiveness of respite care services for people with dementia will be needed first. It is suggested to also include pre-posttest designs without control group in order to explore more potential effects. Second, we also recommend new intervention studies investigating different forms of respite care services most preferably using a comparable control group allowing stronger conclusions to be made.

\section{Conclusion}

Stating which intervention type works best for all caregivers and also indirectly benefits recipients is still impossible due to the complex nature of supporting strategies and the current methodological issues. Yet we can conclude that supporting caregivers is important and effective because, when properly implemented, it has the potential to improve well-being of caregivers and their care-recipients, resulting inevitably in additional benefits for society.

After all, informal caregivers play an increasingly important role in the care process given the current trend toward community-based health care in all health policies to press rising health care costs and to improve quality of life. Policy makers must value and recognize their exceptional role and support them so they can sustain better.

Future research should deal with the methodological limitations of current evidence in this research 
field to draw more clear and unambiguous conclusions. There is also a need to further explore possible effectiveness and cost-effectiveness of respite care.

\section{ACKNOWLEDGMENTS}

This project is funded by the InBev-Baillet Latour fund and the authors declare to have no financial interest in the results of this paper. The funding resources had no role in the development of this paper and in final publication decisions.

Authors' disclosures available online (http://j-alz. com/manuscript-disclosures/15-1011r2).

\section{SUPPLEMENTARY MATERIAL}

The supplementary material is available in the electronic version of this article: http://dx.doi.org/ 10.3233/JAD-151011.

\section{REFERENCES}

[1] Van Mierlo LD, Meiland FJ, Van der Roest HG, Droes RM (2012) Personalised caregiver support: Effectiveness of psychosocial interventions in subgroups of caregivers of people with dementia. Int J Geriatr Psychiatry 27, 1-14.

[2] Boots LM, de Vugt ME, van Knippenberg RJ, Kempen GI, Verhey FR (2014) A systematic review of Internetbased supportive interventions for caregivers of patients with dementia. Int J Geriatr Psychiatry 29, 331-344.

[3] Jensen M, Agbata IN, Canavan M, McCarthy G (2015) Effectiveness of educational interventions for informal caregivers of individuals with dementia residing in the community: Systematic review and meta-analysis of randomised controlled trials. Int J Geriatr Psychiatry 30, 130-143.

[4] Cooke DD, McNally L, Mulligan KT, Harrison MJ, Newman SP (2001) Psychosocial interventions for caregivers of people with dementia: A systematic review. Aging Ment Health 5, 120-135.

[5] Van't Leven N, Prick AE, Groenewoud JG, Roelofs PD, de Lange J, Pot AM (2013) Dyadic interventions for community-dwelling people with dementia and their family caregivers: A systematic review. Int Psychogeriatr 25, 1581-1603.

[6] Thinnes A, Padilla R (2011) Effect of educational and supportive strategies on the ability of caregivers of people with dementia to maintain participation in that role. Am J Occup Ther 65, 541-549.

[7] Grant I, McKibbin CL, Taylor MJ, Mills P, Dimsdale J, Ziegler M, Patterson TL (2003) In-home respite intervention reduces plasma epinephrine in stressed Alzheimer caregivers. Am J Geriatr Psychiatry 11, 62-72.

[8] Gendron M (2001) Baluchon Alzheimer: A Respite Care and Home Support Service for Caregivers of Alzheimer's Patients. The Canadian Alzheimer Disease Review, July 2001, pp. 4-7.
[9] Zank S, Schacke C (2002) Evaluation of geriatric day care units: Effects on patients and caregivers. J Gerontol B Psychol Sci Soc Sci 57, P348-P357.

[10] Epp T (2003) Person-centered dementia care: A vision to be refined. The Canadian Alzheimer Disease Review 5, 14-18.

[11] Thompson CA, Spilsbury K, Hall J, Birks Y, Barnes C, Adamson J (2007) Systematic review of information and support interventions for caregivers of people with dementia. BMC Geriatr 7, 18.

[12] Pinquart M, Sorensen S (2006) Helping caregivers of persons with dementia: Which interventions work and how large are their effects? Int Psychogeriatrics 18, 577-595.

[13] Vernooij-Dassen M, Draskovic I, McCleery J, Downs M (2011) Cognitive reframing for carers of people with dementia. Cochrane Database Syst Rev, CD005318.

[14] Martin-Carrasco M, Ballesteros-Rodriguez J, Dominguez-Panehon AI, Munoz-Hermoso P, GonzalezFraile E (2014) Interventions for caregivers of patients with dementia. Actas Esp Psiquiatr 42, 300-314.

[15] Sorensen S, Duberstein P, Gill D, Pinquart M (2006) Dementia care: Mental health effects, intervention strategies, and clinical implications. Lancet Neurol 5, 961-973.

[16] Hall L, Shelton DA (2012) Occupational therapy for caregivers of people with dementia: A review of the United Kingdom literature. Br J Occup Ther 75, 281-288.

[17] Gaugler JE, Yu F, Krichbaum K, Wyman JF (2009) Predictors of nursing home admission for persons with dementia. Med Care 47, 191-198.

[18] Dodge R, Daly A, Huyton J, Sanders L (2012) The challenge of defining wellbeing. Int J Wellbeing 2, 222-235.

[19] Veenhoven R (2008) Sociological theories of subjective well-being. In The science of well-being, Eid M, Larsen RJ, eds. The Guilford Press, New York, pp. 44-62.

[20] Chappell NL, Reid RC (2002) Burden and well-being among caregivers: Examining the distinction. Gerontologist 42, 772-780.

[21] Stull DE, Kosloski K, Kercher K (1994) Caregiver burden and generic well-being: Opposite sides of the same coin? Gerontologist 34, 88-94.

[22] Gitlin LN, Winter L, Corcoran M, Dennis MP, Schinfeld S, Hauck WW (2003) Effects of the home Environmental Skill-Building Program on the caregiver-care recipient dyad: 6-month outcomes from the Philadelphia REACH initiative. Gerontologist 43, 532-546.

[23] Gavrilova SI, Ferri CP, Mikhaylova N, Sokolova O, Banerjee S, Prince M (2009) Helping carers to care-The 10/66 Dementia Research Group's randomized control trial of a caregiver intervention in Russia. Int J Geriatr Psychiatry 24, 347-354.

[24] Livingston G, Barber J, Rapaport P, Knapp M, Griffin M, King D, Livingston D, Mummery C, Walker Z, Hoe J, Sampson EL, Cooper C (2013) Clinical effectiveness of a manual based coping strategy programme (START, STrAtegies for RelaTives) in promoting the mental health of carers of family members with dementia: Pragmatic randomised controlled trial. BMJ 347, f6276.

[25] Mittelman MS, Haley WE, Clay OJ, Roth DL (2006) Improving caregiver well-being delays nursing home placement of patients with Alzheimer disease. Neurology 67, 1592-1599.

[26] Kurz A, Wagenpfeil S, Hallauer J, Schneider-Schelte H, Jansen S, Grp AS (2010) Evaluation of a brief educational program for dementia carers: The AENEAS Study. Int J Geriatr Psychiatry 25, 861-869. 
[27] Dias A, Dewey ME, D'Souza J, Dhume R, Motghare DD, Shaji KS, Menon R, Prince M, Patel V (2008) The effectiveness of a home care program for supporting caregivers of persons with dementia in developing countries: A randomised controlled trial from Goa, India. PLoS One 3, e2333.

[28] Phung KTT, Waldorff FB, Buss DV, Eckermann A, Keiding N, Rishoj S, Siersma V, Sorensen J, Sogaard R, Sorensen LV, Vogel A, Waldemar G (2013) A three-year follow-up on the efficacy of psychosocial interventions for patients with mild dementia and their caregivers: The multicentre, rater-blinded, randomised Danish Alzheimer Intervention Study (DAISY). BMJ Open 3, e003584.

[29] Nehen HG, Hermann DM (2015) Supporting dementia patients and their caregivers in daily life challenges: Review of physical, cognitive and psychosocial intervention studies. Eur J Neurol 22, 246-250.

[30] Lopez-Hartmann M, Wens J, Verhoeven V, Remmen R (2012) The effect of caregiver support interventions for informal caregivers of community-dwelling frail elderly: A systematic review. Int J Integr Care 12, e133.

[31] Sorensen S, Pinquart M, Duberstein P (2002) How effective are interventions with caregivers? An updated meta-analysis. Gerontologist 42, 356-372.

[32] Schulz R, O'Brien A, Czaja S, Ory M, Norris R, Martire LM, Belle SH, Burgio L, Gitlin L, Coon D, Burns R, Gallagher-Thompson D, Stevens A (2002) Dementia caregiver intervention research: In search of clinical significance. Gerontologist 42, 589-602.

[33] Olazaran J, Reisberg B, Clare L, Cruz I, Pena-Casanova J, del Ser T, Woods B, Beck C, Auer S, Lai C, Spector A, Fazio S, Bond J, Kivipelto M, Brodaty H, Manuel RJ, Collins H, Teri L, Mittelman M, Orrell M, Feldman HH, Muniz R (2010) Nonpharmacological therapies in Alzheimer's disease: A systematic review of efficacy. Dement Geriatr Cogn Disord 30, 161-178.

[34] The Swedish Council on Technology Assessment in Health Care (2008) Chapter 34 in Dementia - caring, ethics, ethnical and economical aspects (172E/3). The Swedish Council on Technology Assessment in Health Care, Stockholm, pp. 317-398.

[35] Voigt-Radloff S, Graff M, Leonhart R, Hull M, Rikkert MO, Vernooij-Dassen M (2011) Why did an effective Dutch complex psycho-social intervention for people with dementia not work in the German healthcare context? Lessons learnt from a process evaluation alongside a multicentre RCT. BMJ Open 1, e000094.

[36] Brodaty H, Green A, Koschera A (2003) Meta-analysis of psychosocial interventions for caregivers of people with dementia. J Am Geriatr Soc 51, 657-664.

[37] Maayan N, Soares-Weiser K, Lee H (2014) Respite care for people with dementia and their carers. Cochrane Database Syst Rev 1, CD004396.

[38] Neville C, Beattie E, Fielding E, MacAndrew M (2015) Literature review: Use of respite by carers of people with dementia. Health Soc Care Community 23, 51-63.

[39] Jeon YH, Brodaty H, Chesterson J (2005) Respite care for caregivers and people with severe mental illness: Literature review. $J$ Adv Nurs 49, 297-306.

[40] Cooper C, Balamurali TBS, Selwood A, Livingston G (2007) A systematic review of intervention studies about anxiety in caregivers of people with dementia. Int J Geriatr Psychiatry 22, 181-188.
[41] Cooper C, Mukadam N, Katona C, Lyketsos CG, Ames D, Rabins PV, Engedal K, Lima C, Blazer D, Teri L, Brodaty H, Livingston G (2012) Systematic review of the effectiveness of non-pharmacological interventions to improve quality of life of people with dementia. Int Psychogeriatrics 24, 856-870.

[42] Spijker A, Vernooij-Dassen M, Vasse E, Adang E, Wollersheim H, Grol R, Verhey F (2008) Effectiveness of nonpharmacological interventions in delaying the institutionalization of patients with dementia: A meta-analysis. $J$ Am Geriatr Soc 56, 1116-1128.

[43] Yin T, Zhou QP, Bashford C (2002) Burden on family members - Caring for frail elderly: A meta-analysis of interventions. Nurs Res 51, 199-208.

[44] McKechnie V, Barker C, Stott J (2014) Effectiveness of computer-mediated interventions for informal carers of people with dementia-a systematic review. Int Psychogeriatrics 26, 1619-1637.

[45] Selwood A, Johnston K, Katona C, Lyketsos C, Livingston G (2007) Systematic review of the effect of psychological interventions on family caregivers of people with dementia. $J$ Affect Disord 101, 75-89.

[46] Downs SH, Black N (1998) The feasibility of creating a checklist for the assessment of the methodological quality both of randomised and non-randomised studies of health care interventions. J Epidemiol Community Health 52, 377-384.

[47] Verhaeghe N, De Maeseneer J, Maes L, Van Heeringen C, Annemans L (2011) Perceptions of mental health nurses and patients about health promotion in mental health care: A literature review. J Psychiatr Ment Health Nurs 18, $487-$ 492.

[48] Lukens EP PhD, McFarlane WR MD (2004) Psychoeducation as evidence-based practice: Considerations for practice, research, and policy. Brief Treatm Crisis Interv 4, 205-225.

[49] Acton GJ, Kang J (2001) Interventions to reduce the burden of caregiving for an adult with dementia: A metaanalysis. Res Nurs Health 24, 349-360.

[50] Mason A, Weatherly H, Spilsbury K, Arksey H, Golder S, Adamson J, Drummond M, Glendinning C (2007) A systematic review of the effectiveness and cost-effectiveness of different models of community-based respite care for frail older people and their carers. Health Technol Assess 11, 1-157.

[51] Graff MJL, Vernooij-Dassen MJM, Thijssen M, Dekker J, Hoefnagels WHL, Rikkert M (2006) Community based occupational therapy for patients with dementia and their care givers: Randomised controlled trial. BMJ 333, 11961199.

[52] Miller PA, Butin D (2000) The role of occupational therapy in dementia - COPE (Caregiver Options for Practical Experiences). Int J Geriatr Psychiatry 15, 86-89.

[53] Gonzalez-Pinto A, Gonzalez C, Enjuto S, Fernandez de Corres B, Lopez P, Palomo J, Gutierrez M, Mosquera F, Perez de Heredia JL (2004) Psychoeducation and cognitive-behavioral therapy in bipolar disorder: An update. Acta Psychiatr Scand 109, 83-90.

[54] Kwok T, Au A, Wong B, Ip I, Mak V, Ho F (2014) Effectiveness of online cognitive behavioral therapy on family caregivers of people with dementia. Clin Interv Aging 9, 631-636.

[55] Vickrey BG, Mittman BS, Connor KI, Pearson ML, Della Penna RD, Ganiats TG, DeMonte RW, Jr., Chodosh J, Cui 
X, Vassar S, Duan N, Lee M (2006) The effect of a disease management intervention on quality and outcomes of dementia care - A randomized, controlled trial. Ann Intern Med 145, 713-726.

[56] Charlesworth G, Shepstone L, Wilson E, Thalanany M, Mugford M, Poland F (2008) Does befriending by trained lay workers improve psychological well-being and quality of life for carers of people with dementia, and at what cost? A randomised controlled trial. Health Technol Assess 12, $1-78$.

[57] Melis RJ, van Eijken MI, van Achterberg T, Teerenstra S, Vernooij-Dassen MJ, van de Lisdonk EH, Rikkert MG (2009) The effect on caregiver burden of a problem-based home visiting programme for frail older people. Age Ageing 38, 542-547.

[58] Winter L, Gitlin LN (2006) Evaluation of a telephonebased support group intervention for female caregivers of community-dwelling individuals with dementia. Am J Alzheimers Dis Other Demen 21, 391-397.

[59] Joling KJ, van Marwijk HW, Smit F, van der Horst HE, Scheltens P, van de Ven PM, Mittelman MS, van Hout HP (2012) Does a family meetings intervention prevent depression and anxiety in family caregivers of dementia patients? A randomized trial. PLoS One 7, e30936.

[60] Tremont G, Davis JD, Bishop DS, Fortinsky RH (2008) Telephone-delivered psychosocial intervention reduces burden in dementia caregivers. Dementia 7, 503-520.

[61] Connell CM, Janevic MR (2009) Effects of a telephonebased exercise intervention for dementia caregiving wives a randomized controlled trial. J Appl Gerontol 28, 171194.

[62] Liddle J, Smith-Conway ER, Baker R, Angwin AJ, Gallois C, Copland DA, Pachana NA, Humphreys MS, Byrne GJ, Chenery HJ (2012) Memory and communication support strategies in dementia: Effect of a training program for informal caregivers. Int Psychogeriatr 24, 1927-1942.

[63] Teri L, Gibbons LE, McCurry SM, Logsdon RG, Buchner DM, Barlow WE, Kukull WA, LaCroix AZ, McCormick W, Larson EB (2003) Exercise plus behavioral management in patients with Alzheimer disease: A randomized controlled trial. JAMA 290, 2015-2022.

[64] Martin-Carrasco M, Franco Martin M, Pelegrin Valero C, Roy Millan P, Iglesias Garcia C, Ros Montalban S, Gobartt Vazquez AL, Pons Piris S, Balana Vilanova M (2009) Effectiveness of a psychoeducational intervention program in the reduction of caregiver burden in Alzheimer's disease patients' caregivers. Int J Geriatr Psychiatry 24, 489-499.

[65] Ducharme FC, Levesque LL, Lachance LM, Kergoat MJ, Legault AJ, Beaudet LM, Zarit SH (2011) "Learning to Become a Family Caregiver" efficacy of an intervention program for caregivers following diagnosis of dementia in a relative. Gerontologist 51, 484-494.

[66] Ducharme F, Lachance L, Levesque L, Zarit SH, Kergoat M-J (2015) Maintaining the potential of a psychoeducational program: Efficacy of a booster session after an intervention offered family caregivers at disclosure of a relative's dementia diagnosis. Aging Ment Health 19, 207-216.

[67] Livingston G, Barber J, Rapaport P, Knapp M, Griffin M, King D, Romeo R, Livingston D, Mummery C, Walker Z, Hoe J, Cooper C (2014) Long-term clinical and cost-effectiveness of psychological intervention for family carers of people with dementia: A single-blind, randomised, controlled trial. Lancet Psychiatry 1, 539-548.

[68] Andrén S, Elmståhl S (2008) Psychosocial intervention for family caregivers of people with dementia reduces caregiver's burden: Development and effect after 6 and 12 months. Scand J Caring Sci 22, 98-109.

[69] Andrén S, Elmstahl S (2008) Effective psychosocial intervention for family caregivers lengthens time elapsed before nursing home placement of individuals with dementia: A five-year follow-up study. Int Psychogeriatr 20, 1177-1192.

[70] Berger G, Bernhardt T, Schramm U, Muller R, LandsiedelAnders S, Peters J, Kratzsch T, Frolich L (2004) No effects of a combination of caregivers support group and memory training/music therapy in dementia patients from a memory clinic population. Int J Geriatr Psychiatry 19, 223-231.

[71] Dahlrup B, Nordell E, Andren S, Elmstahl S (2011) Family caregivers' assessment of symptoms in persons with dementia using the GBS-scale: Differences in rating after psychosocial intervention - an 18-month follow-up study. Clin Interv Aging 6, 9-18.

[72] Hepburn KW, Tornatore J, Center B, Ostwald SW (2001) Dementia family caregiver training: Affecting beliefs about caregiving and caregiver outcomes. $J$ Am Geriatr Soc 49, 450-457.

[73] Ulstein ID, Sandvik L, Wyller TB, Engedal K (2007) A one-year randomized controlled psychosocial intervention study among family carers of dementia patients - Effects on patients and carers. Dement Geriatr Cogn Disord 24, 469-475.

[74] de Rotrou J, Cantegreil I, Faucounau V, Wenisch E, Chausson C, Jegou D, Grabar S, Rigaud AS (2011) Do patients diagnosed with Alzheimer's disease benefit from a psycho-educational programme for family caregivers? A randomised controlled study. Int J Geriatr Psychiatry 26, 833-842.

[75] Martin-Carrasco M, Dominguez-Panchon AI, GonzalezFraile E, Munoz-Hermoso P, Ballesteros J (2014) Effectiveness of a psychoeducational intervention group program in the reduction of the burden experienced by caregivers of patients with dementia: The EDUCA-II Randomized Trial. Alzheimer Dis Assoc Disord 28, 79-87.

[76] Steffen AM (2000) Anger management for dementia caregivers: A preliminary study using video and telephone interventions. Behav Ther 31, 281-299.

[77] Mahoney DF, Tarlow BJ, Jones RN (2003) Effects of an automated telephone support system on caregiver burden and anxiety: Findings from the REACH for TLC intervention study. Gerontologist 43, 556-567.

[78] Drentea P, Clay OJ, Roth DL, Mittelman MS (2006) Predictors of improvement in social support: Five-year effects of a structured intervention for caregivers of spouses with Alzheimer's disease. Social Sci Med 63, 957-967.

[79] Mittelman MS, Roth DL, Haley WE, Zarit SH (2004) Effects of a caregiver intervention on negative caregiver appraisals of behavior problems in patients with Alzheimer's disease: Results of a randomized trial. J Gerontol B Psychol Sci Soc Sci 59, 27-34.

[80] Mittelman MS, Roth DL, Coon DW, Haley WE (2004) Sustained benefit of supportive intervention for depressive symptoms in caregivers of patients with Alzheimer's disease. Am J Psychiatry 161, 850-856. 
[81] Mittelman MS, Roth DL, Clay OJ, Haley WE (2007) Preserving health of Alzheimer caregivers: Impact of a spouse caregiver intervention. Am J Geriatr Psychiatry 15, 780789.

[82] Menn P, Holle R, Kunz S, Donath C, Lauterberg J, Leidl R, Marx P, Mehlig H, Ruckdaschel S, Vollmar HC, Wunder S, Grassel E (2012) Dementia care in the general practice setting: A cluster randomized trial on the effectiveness and cost impact of three management strategies. Value Health 15, 851-859.

[83] Hepburn K, Lewis M, Tornatore J, Sherman CW, Bremer KL (2007) The Savvy Caregiver program: The demonstrated effectiveness of a transportable dementia caregiver psychoeducation program. J Gerontol Nurs 33, 30-36.

[84] Nichols LO, Chang C, Lummus A, Burns R, MartindaleAdams J, Graney MJ, Coon DW, Czaja S (2008) The costeffectiveness of a behavior intervention with caregivers of patients with Alzheimer's disease. J Am Geriatr Soc 56, 413-420.

[85] Eloniemi-Sulkava U, Saarenheimo M, Laakkonen ML, Pietila M, Savikko N, Kautiainen H, Tilvis RS, Pitkala KH (2009) Family care as collaboration: Effectiveness of a multicomponent support program for elderly couples with dementia. Randomized controlled intervention study. J Am Geriatr Soc 57, 2200-2208.

[86] Baumgarten M, Lebel P, Laprise H, Leclerc C, Quinn C (2002) Adult day care for the frail elderly: Outcomes, satisfaction, and cost. J Aging Health 14, 237-259.

[87] Femia EE, Zarit SH, Stephens MA, Greene R (2007) Impact of adult day services on behavioral and psychological symptoms of dementia. Gerontologist 47, 775-788.

[88] Mossello E, Caleri V, Razzi E, Di Bari M, Cantini C, Tonon E, Lopilato E, Marini M, Simoni D, Cavallini MC, Marchionni N, Biagini CA, Masotti G (2008) Day Care for older dementia patients: Favorable effects on behavioral and psychological symptoms and caregiver stress. Int J Geriatr Psychiatry 23, 1066-1072.

[89] Graff MJL, Vernooij-Dassen MJM, Thijssen M, Dekker J, Hoefnagels WHL, OldeRikkert MGM (2007) Effects of community occupational therapy on quality of life, mood, and health status in dementia patients and their caregivers: A randomized controlled trial. J Gerontol A Biol Sci Med Sci 62, 1002-1009.

[90] Gitlin LN, Corcoran M, Winter L, Boyce A, Hauck WW (2001) A randomized, controlled trial of a home environmental intervention: Effect on efficacy and upset in caregivers and on daily function of persons with dementia. Gerontologist 41, 4-14.

[91] Gitlin LN, Hauck WW, Dennis MP, Winter L (2005) Maintenance of effects of the home environmental skill- building program for family caregivers and individuals with Alzheimer's disease and related disorders. J Gerontol A Biol Sci Med Sci 60, 368-374.

[92] Gitlin LN, Winter L, Burke J, Chernett N, Dennis MP, Hauck WW (2008) Tailored activities to manage neuropsychiatric behaviors in persons with dementia and reduce caregiver burden: A randomized pilot study. $\mathrm{Am}$ J Geriatr Psychiatry 16, 229-239.

[93] Gitlin LN, Winter L, Dennis MP, Hodgson N, Hauck WW (2010) Targeting and managing behavioral symptoms in individuals with dementia: A randomized trial of a nonpharmacological intervention. J Am Geriatr Soc 58, 1465-1474.

[94] Nobili A, Riva E, Tettamanti M, Lucca U, Liscio M, Petrucci B, Porro GS (2004) The effect of a structured intervention on Caregivers of patients with dementia and problem behaviors - A randomized controlled pilot study. Alzheimer Dis Assoc Disord 18, 75-82.

[95] Woods RT, Bruce E, Edwards RT, Elvish R, Hoare Z, Hounsome B, Keady J, Moniz-Cook ED, Orgeta V, Orrell M, Rees J, Russell IT (2012) REMCARE: Reminiscence groups for people with dementia and their family caregivers - effectiveness and cost-effectiveness pragmatic multicentre randomised trial. Health Technol Assess 16, 1-116.

[96] Losada A, Marquez-Gonzalez M, Romero-Moreno R (2011) Mechanisms of action of a psychological intervention for dementia caregivers: Effects of behavioral activation and modification of dysfunctional thoughts. Int J Geriatr Psychiatry 26, 1119-1127.

[97] Rodriguez-Sanchez E, Patino-Alonso MC, Mora-Simon S, Gomez-Marcos MA, Perez-Penaranda A, Losada-Baltar A, Garcia-Ortiz L (2013) Effects of a psychological intervention in a primary health care center for caregivers of dependent relatives: A randomized trial. Gerontologist 53, 397-406.

[98] Passoni S, Moroni L, Toraldo A, Mazza MT, Bertolotti G, Vanacore N, Bottini G (2014) Cognitive behavioral group intervention for Alzheimer caregivers. Alzheimer Dis Assoc Disord 28, 275-282.

[99] Smits CHM, de Lange J, Droes R-M, Meiland F, Vernooij-Dassen M, Pot AM (2007) Effects of combined intervention programmes for people with dementia living at home and their caregivers: A systematic review. Int J Geriatr Psychiatry 22, 1181-1193.

[100] Lee H, Cameron M (2004) Respite care for people with dementia and their carers. Cochrane Database Syst Rev, CD004396. 